\title{
ZENTRALSTELLEN FÜR HOCHSCHULWESEN UND WISSENSCHAFTLICHE ZUSAMMENARBEIT
}

\section{International}

UNESCO. United Nations Educational, Scientific and Cultural Organization / Organisation des Nations Unies pour l'Éducation, la Science et la Culture Organización de las Naciones Unidas para la Educación, la Clencia y la Cultura (1946) [7/9, Place de Fontenoy, Paris 7e/France]. - New York Office: [Room 2201, UN Building, 42nd Street at 1st Avenue, New York 17, N.Y./U.S.A.]. - Dir. Gen.: Renè Maheu (France). - P: World Survey of Education; Higher Education in the U.S.S.R.; Higher Education in Europe; Statistical Yearbook; UNESCO Courier; UNESCO Chronicle; International Social Science Journal; Bibliography of Publications Designed to Raise the Standard of Scientific Literature; Bulletin for Libraries; Copyright Bulletin; Museum; Impact of Science on Society.

WUS. World University Service / Entr'aide Universitaire Mondiale $(1920 ; 1950)$ [13, rue Calvin, Genève/Suisse]. - Prés.: Dr. P.H. Qureshi. -- Secr. Gén.: Hans A.H. Dall. - P: WUS in Action; Programme of Action; Annual Report; Reports on Conferences and Seminars.

IBE. International Bureau of Education / Bureau International d'Éducation (1925) [Palais Wilson, 1211 Genève 14/Suisse]. - Dir.: Prof. J. Plaget. P: International Yearbook of Education; Bulletin of the IBE; Reports of Inquiries and National Monographs on Education.

ICPHS. International Council for Philosophy and Humanistic Studies / Conseil International de la Philosophie et des Sciences Humaines (1949). - Headquarter: [Palais des Acadèmies, 1 rue Ducale, Bruxelles/Belgique]. Secretariat: [Maison de l'UNESCO, 6 rue Franklin, Paris 16e/France]. Prés.: Silvio Zavala (Mexico). - P. Bulletin d'Information; Diogènes.

ICSU. International Council of Sclentific Unions / Conseil International des Unions Scientiflques (1931) [7, Via Cornelio Celso, Roma 00161/Italia. Tel. 862555]. - Pres.: Dr. J.M. Harris on (Canada). - Secr. Gen.: Prof. K. Chandrasekharan (India). - P: Year Book of the iscu; ISCU Bulletin.

World Confederation of Organizations of the Teaching Profession (1952) [1330 Massachusetts Avenue, N.W., Washington, D.C. 20005/U.S.A.]. - Pres.: Sir Ronald Gould (Great Britain). - Secr. Gen.: W.G. Carr (U.S.A.). P: Education Panorama; Annual Report; Echo.

International Council on Health, Physical Education and Recreation (1958) [1201 Sixteenth Street, N.W., Washington, D.C. 20006/U.S.A.]

IIE. Institute of International Education (1919) $[809$ United Nations Plaza, New York, N.Y. 10017/U.S.A.]. - Pres.: Kenneth Holland. - P: Annual Report; Handbooks on International Study.

International Institute for Educational Planning (1963) [7 rue Eugène Delacroix, Paris 16e/France]. - A semi-autonomous body established and supported by UNESCO. - Dir.: Philip H. Coombs.

IAU. International Association of Universities / AIU. Association Internationale des Universités / Asoclación Internacional de Universidades (1950). - Permanent Secretariat: [International Universities Bureau / Bureau International des Universités, 6 rue Franklin, 75 Paris $16 \mathrm{e} /$ France]. Prés.: Constantine K. Zurayk (American University of Beirut). 
Vice-Prés.: Jean Roche (Université de Paris). - Secr. Gén.: H.M.R. Keyes. - P: Bulletin of the IAU; International Handbook of Universities; World List / Liste mondiale; Collection of Agreements concerning the Equivalence of University Qualifications / Recueil des accords concernant l'équivalence des titres universitaires; Documents concerning the Equivalence of University Qalifications / Documentation concernant les équivalences des titres universitaires; Cahiers de l'AIU-Papers of the IAU;Studies and Reports / Etudes et Rapports; Joint UNESCO-IAU Research Programme in Higher Education.

AIOSP. Association Internationale d'Orientation Scolaire et Professionelle International Association for Educational and Vocational Guidance (1951) [86 avenue du 10 Septembre, Luxembourg/Luxembourg]. - Prés.: Jean Beaussier (France). - Secr. Gén.: J. Schlitz (Luxembourg). - P: Bulletin d'Information de l'AIOSP.

IAEVI. International Association for Educational and Vocational Information / AIISUP. Association Internationale d'Information Scolaire, Universitaire et Professionelle (1956) [29 rue d'Ulm, Paris 5e/France]. : Prés.: A. Rosier (France). - P: Informations Universitaires et Professionelles Internationales; Colloque et Enquête.

ICUAE. International Congress of University Adult Education / Comité International des Services Universitaires d'Éducation des Adultes (1960) [c/0 Secr. A.A. Liveright, Boston University, 138 Mountfort Street, Brookline, Massachusetts 02 146/U.S.A.]. - Pres.: W.J. McCallion (Canada). P: ICUAE Journal; Occasional Paper; Newsletter; Roster.

International League of Child and Adult Education / Ligue Internationale de l'Enseignement, de l'Éducation et de la Culture Populaire (1947) [3 rue Récamier, Paris 7e/France]. - Prés.: Prof. Sylvain de Coster (Belgique). - Secr. Gén.: Albert J enger (France).

World Education Fellowshlp (1921) [55 Upper Stone Street, Tunbridge Wells, Kent/England]. - Pres.: Prof. K.G. Saiyidain.

IFCU. International Federation of Catholic Universities / FIUC. Fédération Internationale des Universités Catholiques (1949) [ 77 bis, rue de Grenelle, Paris $7 \mathrm{e} /$ France]. - Ancient nom: Foederatio Universitatum Catholicarum. - Prés.: Rev. Theodore Hesburgh (U.S.A.). - P: Annuarium-Catholicarum Universitatum Foederatio; Catalogus Catholicarum Institutorum de Studiis Superioribus; Supplementa Annuarii et Catalogi Seminaria Maiora; Documenta Catholicarum Universitatum Foederationis.

IAUPL. International Association of University Professors and Lecturers Association Internationale des Professeurs et Maîtres de Conference des Universités (1944) [Rozier 6, Ghent/Belgique]. - Prés.: Prof. L.E. Roulet (Suisse). - P: Communication; The Recruitment and Training of University Teachers.

International Federation of Workers' Educational Associations (1947) [Temple House, 27 Portman Square, London W. 1/England]. - Pres.: S.A. Stahre (Sverige).

FIOCES. Fédération Internationale des Organisations de Correspondance et d'Echanges Scolaires / International Federation of Organisations for School Correspondence and Exchange (1929) [29 rue d'Ulm, Paris 5e/France]. Prés.: Prof. O. Vidaeus (Sverige). - Secr. Gén.: J. Damanty (France). - P: Bulletin.

Council on International Educational Exchange (1947) 7777 United Nations Plaza, New York, N.Y. 10017/U.S.A.]. - Additional offices in Paris and Tokyo. - Exec. Dir.: John E. Bow man.

IFUW. International Federation of University Women / FIFDU, Fédération Internationale des Femmes Diplómées des Universités (1919) [17 a King's Road, Sloane Square, London S.W. 3/England]. - Pres.: Mrs. A.K. Hottel. - P: Annual Bulletin; Annual News Letter.

ISMUN. International Student Movement for the United Nations / Mouvement International des Etudiants pour les Nations Unies (1947) [1, Avenue de la Paix, Genève/Suisse]. - Secr. Gén.: Richard Harmston. - P: Bulletin; Documents; Information kits and working papers. 
ISC. International Student Conference / CIE. Conférence Internationale des Etudiants (1950) [c/o Secr. Gen.: Ram Labhaya Lakhina, P.O.Box 36, Leiden/Nederiand]. - P: Information Bulletin; RIC Reports; The Student.

IUS. International Union of Students / UIE. Union Internationale des Étudiants (1946) [Vocelova 3, Praha 2/CSSR]. - Pres.: Zbynék Vokroulicky. Secr. Gen.: Noori Abdul Razzak (Iraq). - P: World Student News; IUS News Service; Young Cinema and Theatre; Sport.

WSCF. World Student Christian Federation / FUACE. Fédération Universelle des Associations Chrétiennes d'Étudiants (1895) [13 rue Calvin, B.P. 206, 1211 Genève 3/Suissel. - Prés.: Philip Potter. - Secr. Gén.: Valdo Galland (Uruguay). - P: Student World; Federation News; Présence.

Pax Romana / International Secretariat for Catholic Students (1921) / International Catholic Movement for Intellectual and Cultural Affairs (1947) [1, Route du Jura, 1701 Fribourg/Suisse]. - Secr. Gén.: P.T. Kuriakose (India). - P: Pax Romana Journal; Vispera; Boletín Ibero-Americano; African Newsletter; Various professional federation publications.

WUJS. World Union of Jewlsh Students / UMEJ. Union Mondiale des Etudiant s Juifs (1924) [c/o Secr. Gén.: Abraham Yehoshua, 18, rue Juliette-Lamber, Paris 17e/France]. - Prés.: Manfred Gerstenfeld.

\section{II. Übernational \\ (regional begrenzt)}

ACU. Association of Commonwealth Universities (1913). - General Office: [36 Gordon Square, London W.C. 1/England. - Tel. 01-3878572]. Branch Office for Commonwealth Scholarships and Appointments: [Marlborough House, Pall Mall, London S.W. 1/England. - Tel. 019393292]. - Formerly names: 1913-1947: Universities Bureau of the British Empire; 1948-1963: AUBC-Association of Universities of the British Commonwealth. - Chairman: Sir Charles Wilson. - Secr. Gen. J. F. Foster. - P: Commonwealth Universities Yearbook; Higher Education in the United Kingdom; United Kingdom Postgraduate Awards; Reports of Proceedings of Congresses of the Universities of the Commonwealth; Reports of Proceedings of Conference of the Home Universities; Radiological Protection in the Universities.

Commonwealth Association of University Teachers (1961) Chairman and Secr.: Prof. J.L. Montrose [c/o Queen's University, Belfast, Northern Ireland/United Kingdom]. - Treasurer: Prof. J.R. Mallory (Canada).

Carnegie Corporation of New York (1911) [589 Fifth Avenue, New York, N.Y. $10017 /$ U.S.A.] - For the advancement of understanding among peoples of U.S. and of the British Overseas Commonwealth. - Acting Pres.: Alan Pifer. - Secr.: Florence Anderson. - P: Annual and Quarterly Reports.

EWA. Education and World Affairs (1962) [522 Fifth Avenue, New York, N.Y. $10036 /$ U.S.A.]. - Three standing committees: The Committee on Education and Human Resource Development (in Africa and Latin America); The Committee on Intercultural Education (stimulating the growth of international education on U.S. Campuses); The Committee on University International Programs (concerned with programs at the larger universities). - Pres.: William W. Marvel. - P: Numerous publications on International Education of Foreign Students.

OES. Overseas Educational Service (1963) [522 Fifth Avenue, New York, N.Y. 10036/U.S.A.]. - Chairman: Herman B. Wells. - Exec. Dir.: John Scot t Everton.

Inter-University Councll for Higher Education Overseas (1946) [33 Bedford Place, London W.C. 1/England]. - Chairman: Lord Fulton. - Secr.: I.C.M. Maxwell.

AUPELF. Association des Universités Partiellement ou Entièrement de Langue Francalse / Association of partially or wholly French-speaking Universities (1961) [c/o Secr. Gén.: Jean-Marc Léger, B.P. 6128, Université de Mon- 
tréal, Montréal 3e/Canada]. - Prés.: H.E. Mohammed El Fasi, Recteur de l'Université Mohammed V, Rabat/Maroc. - Vice-Prés.: Marcel Bouchard; Claude Renard. - Bureau europeen: [173, Boulevard SaintGermain, Paris 6e/France]. - P: La Revue de l'AUPELF; Bulletin de nouvelles brèves; Cahiers de l'AUPELF.

Association Internationale pour la Culture Française à l’Étranger [96 Boulevard Raspail, Paris 6e/France].

Center for Cultural and Technical Interchange between East and West - East West Center (1960) [Honolulu, Hawaii/U.S.A.]. - Chancellor: H. Palfrey Jones.

Direction de la Coopération [15, Rue Boissy-d'Anglas, Paris 8e/France]. Dir.: Jean Auba. - Coopération en matière d'enseignement supérieur entre la France et les États africains d'expression française et la République malgache (Madagascar), ainsi qu'avec Rwanda et le Burundi; aussi avec Fondation de l'Enseignement Supérieur en Afrique Centrale.

Direction de la Coopération avec la Communauté et l'Étranger [55 Rue Saint Dominique, Paris 7e/France]. - Dir.: Stéphane Hessel.

PAN. Pan American Union, Division of Intellectual Cooperation (1890) [Conn Avenue, 17th Street, Washington 6, D.C./U.S.A.]. Intergovernmental organization of the 21 American Republics; central permanent organ and general secretariate of the organization of the American States. Inter-American Cultural Council (1948) [c/o The Secr. Gen.: Pan American Union, Washington 6, D.C./U.S.A.]. - P: Américas.

CHEAR. Councll of Higher Education in the American Republics (1958) [c/0 Secr. Gen.: Kenneth Holland; Institute of International Education, 809 United Nations Plaza, New York, N.Y. 10017/U.S.A.] Supported by the Institute of International Education. - Co-Chairman: Marcel Roche (Venezuela); Grayson Kirk (U.S.A.); Meredith Wilson.

cSUCA. Consejo Superior Universitario Centroamericano / Council of the Confederation of the Universities of Central America / Consell de la Confédération des Universités Centroaméricaines (1948) [Ciudad Universitaria, Rodrigo Facie/Costa Rica, C.A.]. - Pres.: Edmundo Vásquez Martínez (Guatemala). - Secr. Gen.: Edgardo Sevilla I diáquez. - P: Noticias del CSUSA; Repertorio Centroamericano; Boletín informativo de la Secretaría permanente.

Unión de Universidades de América Latina / Union des Universités de l'Amérique Latine / Union of the Universities of Latin America (1949) [Apartado Postal 25232, Ciudad Universitaria, México 20, D.F./México]. - Pres. Prof. Dr. Ignacio González Ginouves (Chile) - Secr. Gen. Dr. Efrén C del Pozo. - P: Universidades; Planes de Estudios de las Universidades latinoamericanas; Escuelas y Carreras profesionales en las Universidades latinoamericanas. Población estudiantil.

ODUCAL. Organización de Universidades Católicas de América Latina / Organization of the Catholic Universities of Latin America (1953) [c/o Secr. Gen.: Fernando Sanhueza Herbage, Universidad Católica de Chile, Avenida Bernardo O'Higgins 340, Casilla 144-D, Santiago de Chile/Chile]. - Pres.: Mgr. Alfredo Silva Santiago, Arzobispo de Petra. - P: Boletin; Documentos.

WUJS. Federación universitaria slonista suramericana [Rio Bamba 1020 , Buenos Aires/Argentina]

UNRWA. United Nations Rellef and Works Agency for Palestine Refugees in the Near East (1950) [Education Department, UNRWA, Beirut]. Educational Services for Universities in the Middle East.

Association of Arab Universities / Union des Universités Arabes (1964). Provisional Bureau: [Dr. Mostafa Kamal Helmy, University of Cairo, Cairo/Arab United Republic].

CAFRAD. Centre Africain de Formation et de Recherches Administratives pour te Devéloppement / African Training and Research Center in Administration for Development (1964) [B.P., 310, 31, Rue de Grenade, Tanger/Maroc. Tél. 164-30]. - Prés.: Dr. Abdelaziz Filali. - Dir.: Ábdel Rahman À bdalla (Khartoum/Sudan). -P: Études Administratives d'Afrique. 
Foundation for Higher Education in Central Africa / Fondation de l'Enseignement Supérieur en Afrique Centrale (1961) [Brazzaville, République du Congo].

West African Examinations Council. - Headquarters Office: [Accra, Ghana]. - Other offices in Lagos/Nigeria; Freetown/Sierra Leone; London/ England. - Conducts G.C.E. Examinations in Ghana, Gambia, Nigeria, and Sierra Leone.

ASAIHL. Association of Southeast Asian Institutions of Higher Learning / Association des Établissements d'Enseignement Supérieur de l'Asie du Sud-Est (1956) [c/o Executive Secr.: H.H. Prof. Prince Prem Purachatra, Ratasastra Building, Chulalongkorn University, Henri Dunant Road, Bangkok/Thailand], - Pres.: Dr. Carlos P. Romulo.

Asian Institute of Educational Planning and Administration (1962) [Ring Road, Indraprastha Estate, New Delhi 1/India]. - Dir.: Dr. K.G. Saiyidain. Executive Dir.: R.K. Kapur. - Sponsorship of UNESCO.

Aslan Regional Institute for School Building Research (1962) [Bandung/ Indonesia]. - Founded under UNESCO sponsorship; serves Asian Member States of UNESCO for school building research and development.

\section{National}

\section{AFGHANISTAN (Doulat-I-Pádscháhi-Ji-Afghánistán)}

Ministry of National Education [Kabul]

National Commission for UNESCO [c/o Ministry of National Education, Kabul]

ALGÉRIE (El Djemhouria El Djazairia Demokratia Echaabia; République Algérienne Démocratique et Populaire)

Ministère de l'Éducation Nationale [Avenue de Pékin, Alger]

Commission Nationale Algérienne pour l'UNESCO [c/o Ministère de l'Ëducation Nationale, Avenue de Pékin, Alger]

Mission Culturelle Française en Algérle [Quartier Arnaud de Vitrolles, Rue Shakespeare, Le Golf, Alger]. -Dir.: M. Rebeyrol.

Office Universitaire et Culturel Français [Lycée Descartes, Avenue de Pékin, Alger]. - Insp. Gén.: Dir. G. Grand.

ANgola (Africa Ocidental Portuguesa)

Direçāo geral do Ensino [Nação]

Sociedade Cultural de Angola [Luanda]. - Pres.: António Alexandre Ca lazans Duarte. - Secr.: Fernando Octávio Portugal Estrela. - P: Cultura.

\section{ARGENTINA (República Argentina)}

Ministerio de Educación y Justicla [Buenos Aires]

IUS. Federación universitaria argentina [Centro de Estudiantes de Medicina, Paraguay 2155, Buenos Aires]

CEIE. Centro de Estadistica e Información Educativa [9 de Julio 1035, Mendoza]. - Dir.: Boris Duplancic.

Comisión nacional de la República Argentina para la UNESCO [Avenida Alvear 1690 , Buenos Aires] 
Instituto Nacional del Profesorado Secundario [c/o Secr.: Floreal Rossi ; José Hernández 2247, Buenos Aires].

IFUW. Federación argentina de Mujeres universitarias [Hippólito Yrigoyen 2038, Buenos Aires]. - Pres.: Dr. M.R. Oliver. - Secr. Intern.: Dr. N.Y. deMontelatici.

WSCF. Movimiento estudiantil cristlano de Argentina [c/o Secr. Gen.: Néstor Raúl Garcia, Cangallo 1644, Buenos Aires]

Pax Romana [Alsina 830, Buenos Aires] Agrupación de Profesionales de la Acción católica argentina Juventud universitaria católica Agrupación de Universitarias de Acción católica

Organización Mundial Universitaria (1953) [Juncal 1258, Buenos Aires]. Dir.: Ariel Fernández Dirube.

ISMUN. Movimiento Argentino de Juventud por Naclones Unidas [c/o Secr. Gen.: Guillermo Giacos, Córdoba 1749, Rosario].

AUSTRALIA (The Commonwealth of Australia)

The Commonwealth Office of Education (1945) [50 Miller Street, North Sydney, N.S.W.]. - Dir.: W.J. Weeden.

Department of Territories (External Affairs) [Canberra, A.C.T.]

The Australian Universities Commission (1959) [c/0 Secr.: D. Dexter, P.O.Box 250, Canberra, A.C.T.]. - Chairman: Sir Leslie Martin.

Australian National Advisory Committee for UNESCO [c/o Commonwealth Office of Education, 50 Miller Street, North Sidney, N.S.W.]

World University Service [c/o Execut. Secr.: David Muschamp; 220 Faraday Street, Carlton, N. 3, Victoria]. - Chairman: Dr. P.J. Fensham.

The Australian Vice-Chancellors' Committee (1920) [c/o Secr.: F.S. Hambly; City Mutual Life Building, Hobart Place, P.O.Box 1142, Canberra, A.C.T.]. - Chairman: Dr. J.A.L. Matheson.

The Commonwealth Scholarships Board (1945). - Chairman: W.J. Weeden.

IAUPL. Federation of Australian University Staff Associations [c/o Secr.: G.W.F.Smith; Harrowgate House, 155-159 Castlereagh Street, Sydney, N.S.W.]

IFUW. Australian Federation of University Women. - Pres.: Miss J. Gilmore [20 Eringa Avenue, Glenunga, South Australia]. - Chairman (International Relations): Mrs. M.B. Johnson.

Council of Adult Education [256 Flinders Street, Melbourne]

ISC. National Union of Australian University Students [52 Story Street, Parkville N. 2, Victoria]

WSCF. Australian Student Christian Movement [c/o Secr. Gen.: Rev. Peter Musgrove; 57 Swanston Street, Melbourne C. 1, Victoria]

Pax Romana. University Catholic Federation of Australia [c/o Secr.: Miss Pat Carroll; St. John's College, Missenden Road, Camperdown, N.S.W.]. Pres.: John Parker.

WUJS. National Union of Australasian Jewish Students [Box 69, The Union, University of Sydney, Sydney, N.S.W.]

\section{BAHAMAS}

Government of the Bahamas. Board of Education [Nassau] 


\section{BARBADOS}

Ministry of Education [St. Michael]

BOLIVIA (República de Bolivia)

Ministerio de Educación y Cultura [La Paz]

Consejo Nacional de Educación [Yanacocha 475, La Paz]

ISC. / IUS. Confeđeración Universitaria Boliviana [c/o Universidad Mayor de San Andrés, Cajón postal 3072, La Paz]

Fundación Universitaria "Simon 1. Patifio" [Comercio 819, Casilla 1265, La Paz]. - Pres.: Dr. Luis Ballivián Saracho. - Secr.'Gen.: Dr. Alberto Salinas López.

Comisión Nacional de la UNESCo [c/o Ministerio de Educación y Cultura, La Paz]

WSCF. Movimiento Estudiantil Cristiano de Bolivia [c/o Secr. Gen.: Geoffrey Driver; Avenida 16 de Julio 1758, La Paz]

Pax Romana. Juventud Universitaria Católica [c/o Pres.: René Lascano; Apartado 1239, Cochabamba]

BOTSWANA (Republic of Botswana)

Department of Education [Mafeking]

BRASIL (Estados Unidos do Brasil)

Ministério da Educação e Cultura [Rua São Clemente, 134, Botafogo, Rio de Janeiro, G.B.]

Conselho Federal da Educaçāo [Palacio da Educação, Rua da Imprensa 16, $5^{\circ}$ Andar, Rio de Janeiro, GB]. - Pres.: Dr. Delindo Couto. - Secr. Ger." Dr. Celso Kelly. - P: Documenta.

CAPES. Coordenação do Aperfelçoamento do Pessoal de Nível Superior [Avenida Marechal Câmara, 210-8 Andar, Rio de Janeiro, GB]. - Pres.: Raimundo Augusto de Castro Moniz de Aragão, Ministro da Educação e Cultura. - Execut. Dir.: Gastao Dias Velloso. - P: CAPES.

IBECC. Instituto Brasileiro da Educa çāo, Ciência e Cultura [UNESCO National Commission, Avenida Presidente Vargas 62, $5^{\circ}$ Andar, Rio de Janeiro, GB]. - Secr. Ger.: Renato Almeida.

Associação Brasileira da Educação (1924) [Avenida Rio Branco 91, $10^{\circ}$ Andar, Caixa Postal 1471, Rio de Janeiro, GB]. - Pres.: Benjamin Albagli. P: Educação.

IFUW. Associaçāo Braslleira de Mulheres Universitárias [Edificio Odeon, Sala 617, Cinelandia, Rio de Janeiro, GB]. - Pres.: Dr. Talitha C. Tudor. Pres. (Relações Internac.): M.N.M. de Oliveira.

ISC. / IUS. Uniāo Nacional dos Estudantes do Brasil [Praia do Flamengo 132, Rio de Janeiro, GB]

WSCF. Uniāo Cristã de Estudantes do Brasil [c/o Secr. Ger.: Edir Cardoso; Caixa postal 416, São Paulo, SP]

Pax Romana [Rua Marechal Francisco Moura No. 63, Apartado 204, Rio de Janeiro, GB. - Rua Capeta Salomao 31, Botafogo, RJ].

BRITISH HONDURAS s. Honduras/British Honduras 
BRUNEI (State of Brunei)

Department of Education [State Education Office, Brunei]

Adult Education Centers [Brunei Town]; [Kuala Belait]; [Seria].

BURMA (Myanma-Nainggan-Daw)

Ministry of Education [Rangoon]

Ministry of Union Culture [Rangoon]

Central Universities Administrative Office [Rangoon]

National Commission for UNESCO [Ministry of Education, Secretariat of the Union of Burma, Rangoon]

IFUW. University Women's Association of Burma. - Pres.: Dr. Thin Kyi [3 Short Road, University Estate, Rangoon]. - Pres. (Internat. Relations): Daw Mya Than Nu.

IUS. All Burma Federation of Student Unions [Rangoon]

WSCF. Student Christian Movement of Burma [c/o Secr. Gen.: Arthur Ko Lay; 601 Prome Road, Kamayut P.O., Rangoon]

Pax Romana. Rangoon University Catholic Students Association [64 Inya Road, University P.O., Rangoon]

BURUNDI (République du Burundi)

Ministère de l'Éducation Nationale et de la Culture [Bujumbura]

Commission Nationale du Burundi pour l'UNEsCo [B.P. 1990, Bujumbura]

IAUPL. Unirundi [B.P. 1320, Usumbura].

ISC. / IUS. Union Nationale des Etudiants Burundi [B.P. 245, Bujumbura]

wus. Comité Rundi de l'Entr'aide Universitaite Mondiale [c/o Université officielle de Bujumbura, B.P. 1550, Bujumbura]. - Prés.: Henri-Philippe Cart.

Pax Romana. Association des Etudiants Catholiques [c/o Université officielle de Bujumbura, B.P. 1550, Bujumbura]

\section{CANADA}

Ministère de l'Éducation [Hotel du Gouvernement, Québec 4]

The Department of the Secretary of State [Ottawa, Ontario]

Education Division of the Dominion Bureau of Statistics [c/o the Chief of Higher Education Section, Ottawa, Ontario]

Department of Education (for the Province of Alberta) [Edmonton].

Department of Education (for the Province of British Columbia) [Victoria, Brit. Columbia].

Department of Education (for the Province of Manitoba) [Winnipeg, Manitoba].

Department of Education (for the Province of New Brunswick) [Moncton, New Brunswick]

Department of Education (for the Province of Newfoundland) [St. Johns, Newfoundland]

Department of Education (for the Province of Nova Scotia) [Halifax, Nova Scotia]. 
Ministry of University Affairs (1946; for the Province of Ontario) [Ottawa, Ontario].

Department of Education (for the Province of Prince Edward Island) [Charlottetown, Prince Edward Island].

Department of Education (for the Province of Quebec) [Quebec, P.Q.].

Department of Education (for the Province of Saskatchewan) [Regina, Saskatchewan]

Canadian National Commission for UNESCO [140 Wellington Street, Ottawa 4, Ontario]

World University Service [328 Adelaide Street West, Toronto 2 B, Ontario]. Chairman: Napoleon Leblanc. - Secr. Gen.: Douglas Mayer.

Association of Universities and Colleges of Canada [c/o Execut, Dir.: Dr. G.C. And rew; 12th floor, 151 Slater Street, Ottawa 4, Ontario]. - Formerly named: the National Conference of Canadian Universities and Colleges and the Canadian Universities Foundation. - Chairman: Mgr. L.A. Vachon.

Association of Atlantic Universities (1964) [Halifax].

Committee of Presidents of Universities of Ontario (1962) [Ottawa, Ontario]

Conférence des Recteurs et des Principaux des Universités du Québec (1964) [Québec, P.Q.]

Canadian Education Association (1891) [c/o the Execut. Secr.: F.K. S tewart 151 Bloor Street West, Toronto 5, Ontario]. - P: Canadian Education and Research Digest; News Letter.

Canadian University Service Overseas / Service Universitaire Canadien OutreMer [75 Albert Street, Ottawa 4, Ontario].

IAUPL. Canadian Association of University Teachers [77, Metcalfe Street, Ottawa, Ontario]

Canadian Association for Adult Education [21-33 Sultan Street, Toronto 5]. Dir.: Alan Thomas. - P: Food for Thougt; Continuous Learning.

IFUW. Canadian Federation of University Women $[29$ Edgedale Road, St. Catharines, Ontario]. - Pres.: Mrs. M.J. Sabia. - Pres. (Internat. Relations): Edith $M$. Woodsworth.

ISC. Canadian Union of Students / Union Canadienne des Etudiants [45 Rideau Street, Ottawa, Ontario].

ISMUN. Student United Nations Association of Canada / Association Canadienne des Étudiants pour les Nations Unies [278 O'Connor Street, Ottawa 4, Ontario]

IUS. / ISC. Union Générale des Étudiants du Québec [1238 est, Boulevard StJoseph, Montréal 34, Québec]

WSCF. Student Christian Movement of Canada / Association Chrétienne des Etudiants Canadiens [1139 Bay Street, Toronto 5, Ontario]

Pax Romana [281 Echo Drive, Ottawa, Ontario]. - [3484 Peel Street, Montréal, Québec].

CAMBODiA (Préah Réach Ana Chak Kampuchéa)

Ministère de 1'Education Nationale [Phnom-Penh].

Commission Nationale Cambodgienne pour l'UNESCO [270, Moha Vithei Préah Bat Monivong, Phnom-Penh].

Pax Romana [c/o Rev. Paul Tep I m, Evéché, Phnom-Penh].

CAMEROUN (République Fédérale du Cameroun)

Ministère de l'Écucation, de la Jeunesse et de la Culture [Yaoundé] 
Commission Nationale de la République Fédérale du Cameroun [B.P. 1061, Yaoundé]

World University Service [c/o M.A. Pellegrin-Hardorff; Université Fédérale du Cameroun, B.P. 337, Yaoundé]

WSCF. Mouvement des Elèves Chrétiens du Cameroun [c/o Secr. Gén.: Maurice Matong; B.P. 89 , Douala]

\section{CENTRAFRICAINE (République Centrafricaine)}

Ministère de l'Éducation Nationale, de la Jeunesse et des Sports [Inspection Académique, B.P. 791, Bangui]

Commission Nationale de la République Centrafricaine pour l'UNESCo [c/o Ministère de l'Éducation Nationale, B.P. 791, Bangui]

\section{CEYLON (Sri Lanka)}

Ministry of Education [Malay Street, Colombo 2]

National Council of Higher Education [202 Bauddaloka Matawa, Colombo 7]. - Chairman: Dr. G.P. Malalasekara. - Secr.: Deryck Aluwihare

Ceylonese National Commission for UNESCO [Ministry of Education, Malay Street, Colombo 2]

World University Service [c/o Health Centre, University Park, Peradeniya]. Chairman: H.P.A. Wijetunge. - Execut. Secr.: Hema Dassanayake.

ISC. Ceylon Unlversity Students' Federation [University of Ceylon, Thurstan Road, Colombo 3]

IUS. Ceylon National Union of Students [292 Galle Road, Colombo 3]

IFUW. Ceylon Federation of University Women [124 Park Road, Colombo 5]. - Pres.: Miss D. Perera. - Chairman (Internat. Relations): Miss C. de Zoysa.

WSCF. Student Christian Movement of Ceylon [SCM Office, YMCA, Colombo 1]

Pax Romana [Aquinas University College, Borella, Colombo 8.

\section{CHILE (República de Chile)}

Ministerio de Educación Pública [Santiago de Chile]

Comisión Nacional de la UNESCo en Chile (Comisión Chilena de Cooperación con la UNESCO) [Mac-Iver 764, Departamento 63, Santiago de Chile]

WUS. Servicio Universitario Chileno [Miguel Claro 1460, Clasificador 1056, Cor. 9, Santiago de Chile]. - Pres.: Huan Gómez Millas. - Secr.: Jaimé Lavados.

Consejo de Rectores de Universidades Chllenas [Moneda 673, Santiago de Chile]. - Pres.: Eugenio González R. - Secr. Gen.: Horacio Serrano P.

Asociación de Educación Nacional [Santiago de Chile]. - Pres.: Dr. Carlos Fernandéz Peña. - Secr. Gen.: Alvaro Rivera M.

ISC. / IUS. Unión de Federaciones Universitarias de Chile [Estado 115, Of. 704, Santiago de Chile]

ISMUN. Asociación Chilena de las Naciones Unidadas [Monjilas 689, Casilla 4001, Of. 46, Santiago de Chile]. - Pres.: C.A. Martinez S.

WSCF. Movimiento Estudiantil Cristiano de Chile [Alameda 723, Casilla 13599, Santiago de Chile]. - Secr. Gen.: Adolfo Covarrubias.

Pax Romana [Villavicencio 337, Santiago de Chile] 


\section{CHINA}

HONG KONG

Education Department [Government of Hong Kong]

Hong Kong Council for Educational Research (1961) [c/o University of Hong Kong]. - Chairman: Prof. Norman K. Henderson.

World University Service [Chung Chi College, Ma Liu Shui, New Territories, Hong Kong], - Chairman: Lyon Lee. - Secr.: Dr. Phillip S.Y. Sun.

IFUW. Hong Kong Association of University Women [University of Hong Kong, Bonham Road, Hong Kong]. - President: Mrs. C.E. Hughes.

WSCF. Student Christian Movement of Hong Kong [23 Waterloo Road, Kowloon, Hong Kongl. - Secr. Gen.: Chiang Pa o Lin.

Pax Romana [16th floor, Grand Building, Connaught Road Central, Hong Kong. - 17 A Sunchun Street, 5/F Tai Hange, Hong Kong]

\section{PEOPLE'S REPUBLIC OF CHINA}

(Dschung-Hua Jen-Min Gung-Ho Guo)

Ministry of Education [Peking, Hopeh]

IUS. All-China Student's Federation [3 Yu Ho Chiao, Peking]

WSCF. The Chinese Student Christian Movement [National YMCA, 131 Huchiu Lu, Shanghai, Kiangsu]

\section{TAIWAN}

Ministry of Education [Taipei, Taiwan]

Chinese National Commission for UNESCO [45 Nan-hai, Road Taipei, Taiwan]

Pax Romana [College of Liberal Arts, Fu-Jen Catholic University Hsinchuang, Taipei, Taiwan]

\section{Colombia (República de Colombia)}

Ministerio de Educación Nacional [Bogotá]

Comisión Nacional Colombiana de Cooperación con la UNEsco [Edificio de los Ministerios, Oficina no. 433, Bogotá]

Asociación Colombiana de Universidades - Fondo Universitario Nacional (1957) [Calle 13 No. 5-33, Carrera 3a-A, No. 18-24, Bogotá]

IUS. Federación Universitaria Nacional [Apartado Nacional 7503, Cuan No. 12110 , Bogotá]

WSCF. Movimiento Estudiantil Cristiano [Apartado 101, Bogotá]

Pax Romana [Calle 16 No. 4-75/79, Apartado Aéreo 11966 , Bogotá. - Avenida 5 A. B No. $22 \mathrm{~N}-45$, Cali]

\section{CONGO}

\section{RÉPUBLIQUE DEMOCRATIQUE DU CONGO (Kinshasa)}

Ministère de l'Éducation Nationale et des Affaires Culturelles [Kinshasa]

WUS. Entr'aide Universitaire Mondiale. - Comités locaux: [c/o Jean-Félix Koli; Université Lovanium, B.P. 800, Kinshasa XI]. - [c/0 Université Officielle du Congo, B.P. 1825, Lubumbashi] 
IUS. Union Générale des Etudiants du Congo [B.P. 5171, Kinshasa X]

WSCF. Union des Étudiants Chrétiens au Congo [B.P. 3094, Kinshasa]. Secr. Gén.: David Katshimuka.

Fédération Congolaise de Pax Romana [c/o Prés.: Isidore Ndaywell, Université Lovanium, B.P. 121, Kinshasa XI]

\section{RÉPUBLIQUE DU CONGO (Brazzaville)}

Direction Générale de l'Enseignement [Ministère de l'Éducation Nationale, B.P. 2078, Brazzaville]

Commission Nationale du Congo pour l'UNESCo [B.P. 493, Brazzaville]

Comité Préparatoire de l'Entr'alde Universitaire Mondlale [B.P. 69, Brazzaville]

Foundation for Higher Education in Central Africa (s. Zentralstellen II)

COSTA RICA (República de Costa Rica)

Ministerio de Educación Pública [San José]

Comisión Costarricense de Cooperación con la UNESCo [c/o Ministerio de Educación Pública, San José]

ISC. Federación de Estudiantes Universitarios de Costa Rica [Apartado 30, Ciudad Universitaria, San José]

WSCF. Movimiento Estudiantil Cristiano de Costa Rica [c/o el Secretario general, Apartado 858, San José]

Pax Romana [Apartado 2715, San José]

\section{CôTE D'IVorRe (République de la Côte d'Ivoire)}

Ministère de l'Éducation Nationale [Abidjan]

Commission Nationale de la Côte d'Ivoire pour l'UNESco [c/o Ministère de l'Éducation Nationale, Abidjan]

Entr'aide Universitaire Mondiale [c/o Université d'Abidjan, Cocody, Abidjan]

ISC. Union Nationale des Etudiants et Elèves de Côte d'Ivoire [B.P. 8018, Cocody, Abidjan]

WSCF. Association Chrétienne d'Étudiants Protestants de Côte d'Ivoire [B.P. 8005, Cocody, Abidjan]. - Sécr. Gén.: Nandjui Dogbo Simon.

Pax Romana. Centre Cathollque des Étudiants d'Abidjan [B.P. 8015, Cocody, Abidjan]. - Pres.: Jean Brizova.

\section{CUBA (República de Cuba)}

Ministerio de Educación [Ciudad Libertad, La Habana]

Comisión Nacional Cubana de la UNESco [Avenida Kohly 151, Nuevo Vedado, La Habana]

ISE. Instituto de Superación Educacional [Ciudad Libertad, Marianao, La Habana]. - Dir.: Dra. María Luisa Rodriguez Cólombié.

IUS. Federación Estudiantil Universitaria [c/o Universidad de La Habana, La Habana]

ISMUN. United Nations Student Association of Cuba [c/o Asociación Cubana de las Naciones Unidas, Calle $\mathrm{J}, \mathrm{n}^{\circ} 514, \mathrm{e} / 23$ y 25 , Vedado, La Habana]. Pres.: Antonio Hernandez Garcia. 
WSCF. Movimiento Estudiantil Cristiano de Cuba [c/o Secr. Gen.: Rodolfo Juarez; K 502, Vedado, La Habana]

DAHOMEY (République du Dahomey)

Ministère de l'Éducation Nationale, de la Jeunesse et des Sports [Porto Novo] Commission Nationale Dahoméenne pour l'UNESCo [c/o Ministère de l'Éducation Nationale, Cotonou]

WSCF. Fédération Dahoméenne des Associations Chrétiennes d'Étudiants [B.P. 34, Cotonou]./- Secr. Gén.: Samuel Assogba.

DOMINICAN REPUBLIC s. República Dominicana

ECUADOR (República del Ecuador)

Ministerio de Educación, Bellas Artes y Cultos [Santo Domingo]

Comisión Nacional Dominicana de la UNESco [c/o Secretaria de Estado de Educación y Bellas Artes, Santo Domingo]

Instituto de Asuntos Inter-Americanos: División de Educación (1942) [Avenida Colón 1717, Quito]. - Dir.: Dr. Lea T. Hearn.

IUS. Federación de Estudiantes Universitarios del Ecuador [Venezuela 1231, Quito]

Pax Romana [Apartado 523, Quito. - Apartado 1049, Quito]

EGYPT s. United Arab Republic

EL SALVADOR (República de El Salvador)

Ministerio de Educación [6a Calle Oriente No. 435, San Salvador]

Comisión Nacional de la Cooperación con la UNESCo [c/o Ministerio de Educación, 6 a Calle Oriente No. 435, San Salvador]

IUS. Asociación General de Estudiantes Universitarios Salvadoreños [Apartado postal 400, San Salvador]

IFUW. Asociación de Mujeres Universitarias de El Salvador [Apartado postal 1293, San Salvador]. - Pres.: Dr. M.G. de Mena Guerrero. - Pres.: (Relaciones Intern.): Dr. A.T. Valiente.

WSCF. Movimiento Estudlantil Cristiano de El Salvador [c/o Secr. Gen.: Guillermo Melgar; Avenida Cuscatlán 528, San Salvador]

Pax Romana [Apartado postal 112, San Salvador]

ETHIOPIA (Jeitiopia Neguse Negest Mengist)

Ministry of Education [Addis Ababa]

Ethiopian National Commission for UNESCo [c/o Ministry of Education, Addis Ababa]

World University Service [c/o Haile Sellassie I University, P.O.Box 1176, Addis Ababa]

ISC. / IUS. National Union of Ethiopian University Students [P.O.Box 3517, Addis Ababa]

WSCF. Haimonate Abew Ethiopian Students Association [c/o Gen. Secr.: Tsegaye Habte; P.O.Box 2189, Addis Ababa] 
Pax Romana. Kessate Berhane Collegiate Association [c/o University College, P.O.Box 399, Addis Ababa]. - Pres.: Ato Amha Tsiyon Domenico.

FIJI (Fiji Islands)

Department of Education [Suva]

WSCF. Fiji Student Christian Movement [c/o YWCA, Suva]

GABON (République du Gabon)

Ministère de l'Éducation Nationale [Libreville]

Commission Nationale Gabonaise pour l'UNESCO [c/o Ministère de l'Éducation Nationale, B.P. 813 , Libreville]

GHANA (Republic of Ghana)

Ministry of Education [Higher Education Division, P.O.Box M. 28, Accra]

Ghana National Commission for UNESCo [c/o Ministry of Education, P.O. Box M. 45, Accra]

African Bureau of Educational Documentation (1961) [Accra]. - UNESCOassisted.

West African Examinations Council (s. Zentralstellen II)

World University Service $[66$ Independence Hall, University of Science and Technology, Kumasi]. - Chairman: Dr. K. Opoku-Owusu. - Secr.: K.A. Ohene-Manu.

ISC. National Union of Ghana Students [University of Ghana, Legon, Accra]

IUS. Ghana National Students' Organization [University of Ghana, Commonwealth Hall, Legon, Accra]

ISMUN. United Nations Student Association of Ghana [Mensah Sarbah Hall, University of Ghana, Legon, Accra]

WSCF. Student Christian Movement of Ghana [c/o Adisadel College, Cape Coast]

Pax Romana [c/o Department of Social Development, P.O.Box 778, Accra. 110 , Independence Hall, University of Science and Technology, Kumasi]

GUAM (Pacific Territory of the U.S.A.)

Department of Education [Agaña Heights]

GUATEMALA (República de Guatemala)

Ministerio de Educación Pública [6a Avenida 1-30, Zona 2, Guatemala]

Instituto Indigenista Nacional [c/o Ministerio de Educación Pública, 6a Avenida 1-30, Zona 2, Guatemala]. - Dir.: Epaminondas Quintana. - P: Boletín; Guatemala Indigeña.

Comisión Guatemalteca de Cooperación con la UNESco [6a Calle 9-27, Guatemala 1]

WUS. Servicio Universitario Mundial [c/o Secr. Gen.: Fernando Andrade; 2a Calle 7-46, Guatemala 9]

ISC. Asociación de Estudiantes Universitarios [10a Avenida "A" 5-40, Guatemala 1] 
WSCF. Asociación Cristiana de Universitarios [c/o Centro Estudiantil Evangélico, Avenida Siméon Cañas 7-13, Guatemala 2]

Pax Romana [10 Avenida 10-51, Guatemala 1]

$$
\text { GUINÉE (République de Guinée) }
$$

Ministère de l'Éducation Nationale [Conakry]

Commission Nationale Guinéenne pour l'UNESCo [c/o Ministère de l'Éducation Nationale, Conakry]

\section{GUYANA}

Ministry of Education and Race Relations [P.O.Box 63, Georgetown]

IUS. Student Councll of Progressive Youth Organisation [41 Robb Street, Lacytown, Georgetown]

HAITI (République d'Haĩti)

Direction Générale de l'Éducation Nationale [Port-au-Prince]

Société d'Études Scientifiques (1937) [Port-au-Prince]. - Prés.: Camille Hérisson.

Commission Nationale Haitienne de Coopération avec l'UNESco [Cité de l'Exposition, Avenue Marie-Jeanne, Port-au-Prince]

Pax Romana [c/o R.P. Antoine Gisler, Archevéché de Port-au-Prince, Port-au-Prince]

HAUTE-VOLTA (République de Haute-Volta)

Ministere de l’Éducation Nationale [Ouagadougou]

Commission Nationale de la République de Haute-Volta pour l'UNESCO [Ministère de l'Éducation Nationale, Ouagadougou]

\section{HONDURAS / BRITISH HONDURAS}

Department of Education [Belize City]

The British Council [4 Albert Street, P.O.Box 331, Belize City]

\section{HONDURAS / REPÚBLICA DE HONDURAS}

MInisterio de Educación Pública [Tegucigalpa, D.C.]

Comislón Nacional de la UNESCO en Honduras [c/o Ministerio de Educación Pública, Tegucigalpa, D.C.]

WUS. Servicio Universitario Mundial [c/o Oficina de Planeamiento de la Universidad Nacional Autónoma de Honduras, Tegucigalpa, D.C.]. Pres.: Arturo Quesada. - Secr. Gen.: Roberto Suazo Tomé.

ISC. Federación de Estudiantes Universitarios de Honduras [Apartado 427, Tegucigalpa, D.C.]

Pax Romana. Acción Católica Universitaria de Honduras [c/o R.P. Juan Antonio Vegas; Apartado postal 489, Tegucigalpa, D.C.]. - Pres.: G.A Landaverde. 
INDIA (Bhārat Juktarashtra)

Ministry of Education [Government of India, Theatre Communication Barracks, Connaught Circus, New Delhi 1]

Inter-University Board of India and Ceylon (1925) [c/o Secr.: Dr. Amrik Singh; Rouse Avenue, New Delhi 1]. - P: The Handbook of Indian Universities; University News; Report.

University Grants Commission (1953) [Bahadus Shah Zafar Marg, Mathura Road, New Delhi 1]. - Chairman: Dr. D.S. Kothari. - Secr.: K.L. Joshi.

Indian National Commission for Cooperation with UNESCO [Ministry of Education, Government of India, New Delhi 3]

World University Service [c/o Execut. Secr.: V.N. Thiagarajan; F 14/21, Model Town, New Delhi 9]. - Chairman: Dr. C.D. Deshmukh.

The Indian Council for Cultural Relations (1950) [Azad Bhavan, Indraprashta Estate, New Delhi 1] Pres.: M.C. Chagla. - Secr.: Inam Rahman. P: Indo-Asian Culture; Cultural News from India; Thaquafat-ul-Hind; I.C.C.R Newsletter.

Indian Institute for Educational and Cultural Co-operation [N.M. Wadia Trust Buildings, 22 D Parsi Bazar Street, Bombay]. - Pres.: H.E. Mr. Sri Prakasa, Governor of Maharashtra.

The Indian Institute of World Culture [6 Shri B.P. Wadia Road, Basavangudi, Bangalore 4]. - Pres.: Sophia W ad i a. - P: Annual Reports; Transactions.

National Council of Educational Research and Training (1963) [38 a Friends Colony, Mathura Road, New Delhi 14]. - Dir.: Dr. T.A. Koshy.

National Fundamental Education Centre (1956) [c/o the National Council of Educational Research and Training, 38 a Friends Colony, Mathura Road, New Delhi 14]. - Dir.: Dr. T.A. Koshy. - Ass. Dir.: H.P. Saksena. P: Reports; NFEC-Bulletin.

National Council of Education, Bengal (1906) [Calcutta 32] Pres.: P.C. Sen, Chief Minister of West Bengal. - Secr.: A.N. Haldar.

IAUPL. All India Federation of University and College Teachers' Organizations [c/o Tilak Dhari College, Jaunpur]. - Secr. Gen.: Prof. H.N. Singh.

Federation of Central Universities Teachers' Associations [Department of Urdu, Aligarh Muslim University, Aligarh]

Indian Adult Education Association (1939) [Indraprashta Marg, New Delhi]. Pres.: Dr. Mohan Sinha Mehta. - Secr.: S.C. Dutta. - P: Indian Journal of Adult Education; Proudh Shiksha; Kamgaar Shiksha.

IFUW. Federation of University Women Indians' Associations [6 Bhagwan Das Road, New Delhi 1]. - Pres.: L.N. Menon. - Chairman (Internat. Relations): Miss H.K. Singh.

ISC. National Council of University Students of India [F-13 South Extension, Part 1, New Delhi 3]

IUS. All India Students' Federation [923, Faiz Road, Karol Bagh, New Delhi] WSCF. Student Christian Movement of India [2-E Mission Road, Bangalore 2]

Pax Romana [c/o St. Xavier's College, Cruikshank Road, Bombay 1. - Loyola College, Madras 34]

ISMUN. United Nations Student Association of India [c/o Secr. Gen.: R.A. Mirza; 103 Purasawalkam High Road, Madras 10]

INDONESIA (Republik Indonesia)

Pendidikan Dasar Dan Kebudajan (Ministry of Basic Education and Culture) [Djakarta] 
Perguruan Tinggi dan IImu Pengetahuan (Ministry of Higher Education and Science) [Djakarta]

Indonesian National Commission for UNESCO [c/0 Biro Hubungan Luar Negeri/UNESCO, Medan Merdeka Timur 14, Djakarta]

Aslan Regional Institute for School Building Research (s. Zentralstellen II)

Jajasan Kerdja-Sama Kebudajaan (Institute for Indonesian Culture) [Djalan Gadjah Mada 13, Bandung]

World University Service [Djalan Kramat 6/37, Djakarta]. - Chairman: R. Slamet Iman Santoso. - Secr. Gen.: Koesnadi Hardjasoemantri.

IUS. Perserikatan Perhimpunan 2 Mahasiswa Indonesla [Djalan Veteran $7 \mathrm{c}$, Djakarta]

IFUW. Perhimpunan Wanita Universitas di Indonesia [c/o Djalan Irian 11, Djakarta]

WSCF. Gerakan Mahasiswa Kristen Indonesla [Salemba 10, Flat 21, Djakarta IV/S]

Pax Romana (Djalan Dr. Sam Ratulangi 1, Djakarta II/16]

IRAN (Keshvaré Shahanshahiyé Irân)

Ministère de l'Éducation [c/o Départment de l'Enseignement Supérieur, Téhéran]

Commission Nationale Iranienne pour l'UNESCO [Avenue du Musée, Téhéran]

ISMUN. Mouvement des Etudiants Iraniens pour les Nations Unies [c/0 Faculté de Droit, Téhéran]

WSCF. Dasste Daneshjouian Massihi (Groupe Universitaire des Étudiants Chrétiens) [c/o R.P.V. Galustian, P.O.Box 2634, Téhéran]

WUJS. Iranlan Jewish Students Organization [39, Rue Heravi, Avenue Pahlavi, Téhéran]

IRAQ (Al Jumhurya al 'Iraqia; Republic Iraq)

Ministry of Education [Baghdad]

Iraql National Commission [c/o Ministry of Education, Baghdad]

ISRAEL (Medinat Israel; State of Israel)

Ministry of Education and Culture [ 34 Shivtey Israel Street, Jerusalem] Council for Higher Education (1958) [34 Shivtey Israel Street, Jerusalem]

Israel National Comission for UNESCo [c/o Ministry of Education and Culture, 34 Shivtey Israel Street, Jerusalem]

World University Service [P.O.Box 1184, Jerusalem]. - Chairman: Ernst D. Bergman. - Secr.: Dan Bitan.

IAUPL. Association of Technion Professors and Lecturers [P.O.Box 4910, Haifa]. - Pres.: Prof. Evyatar.

The Hebrew University of Jerusalem Staff Association [Sherman Building, Jerusalem]

IFUW. Israel Association of University Women [17 Ben Maimon Avenue, Jerusalem]. - Pres.: Dr. H. Gelber.

ISC/WUJS. National Union of Israeli Students [P.O.Box 1184, Jerusalem]

ISMUN. Israel Student Association for the United Nations [P.O.Box 1184, University Campus, Jerusalem] 
Pax Romana. Catholic Students League of Israel [P.O.Box 99, Catholic Seminary, Nazareth]. - Pres.: Ibrahim Tannous.

\title{
IVORY COAST s. Cóte d'Ivoire
}

\begin{abstract}
JAMAICA
Ministry of Education [5 South Race Course, P.O.Box 498, Kingston]

Jamaican National Commission for UNESCo [6 Leinster Road, P.O.Box 202, Kingston 5]

WSCF. Student Christian Movement of Jamaica [P.O.Box 154, Kingston 8]

Pax Romana. Catholic Students Club [c/o Chancellor Hall, University of the West Indies, Mona, Kingston 7]. - Pres.: C.B. Dennis.
\end{abstract}

\section{JAPAN (Nihon-Koku; Nippon)}

Ministry of Education [4 Kasumigaseki, Chiyoda-ku, Tokyo]

Chuo Kyoiku Shingikal (Central Council for Education) [The Ministry of Education, 4 Kasumigaseki, Chiyoda-ku, Tokyo]. - Chairman: Dr. Tatsuo Morito.

Japanese National Commission for UNESCO [c/o Ministry of Education, 4 Kasumigaseki, Chiyoda-ku, Tokyo]

World University Service of Japan [448 Kyodo Setagaya, Tokyo]. - Chairman: Dr. Nobumoto Oh ama. - Execut. Secr.: Makoto Fuj ita.

Kokuritsu Daigaku Kyokai (National University Association of Japan) [c/o Tokyo Daigaku, Hongo 7-3-1, Bunkyo-ku, Tokyo]. - Pres.: Kazuo Okochi.

Koritsu Daigaku Kyokai (Association of Public Local Government Universities) [Nagoya Shiritsu Daigaku, No. 1 Kawasumi, Mizuho-cho, Mizuho-ku, Nagoya]. - Pres.: Ryutaroti Hagino.

Nihon Shiritsu Daigaku Kyokai (Association of Private Universities in Japan) [Shigaku-Kaikan, No. 4, 4-chome, Kudan, Chiyoda-ku, Tokyo]. - Pres.: K. Hiekata. - P: The Kyoiku Gakujustu; Bulletins.

Daigaku Kijun Kyokai (Japanese University Accreditation Association) [20-3 Honshio-cho, Shinjuku-ku, Tokyo]. - Pres.: Takashi Oizumi.

IAUPL. Zenkoku Daigaku Kyoju Rengo (University Professors' Association of Japan) [University of Tokyo, 3,7-chome Hongo, Bunkyo-ku, Tokyo]. - Pres.: Prof. Shokei Takamura.

Nippon Ikuekal (Japan Scholarship Foundation) [42 Ichigaya - Honmuracho, Shinjuku-ku, Tokyo]. - Pres.: Dr. Tatsuo Morito.

IFUW. Japanese Association of University Women [c/o Oinkaikan, 35 Otsukamachi, Bunkyo-ku, Tokyo]. - Pres.: Mrs. K. Kit a mu ra.

IUS. Zengakuren, All Japan Federation of Student Autonomies [c/o Hirota Building, 2-10 Kanda Jinbo-cho, Chiyoda-ku, Tokyo]

WSCF. Student Department, National Committee of YMCAs of Japan [7, 2chome, Fujimi Cho, Chiyoda-ku, Tokyo]

Pax Romana [Shinsei Kaikan, 33 Shinanomachi, Shinjuku-ku, Tokyo]

ISMUN. United Nations Student Association of Japan [2nd Kowa Building, $\mathrm{n}^{\circ}$ 30, Shiba-Shinbashi, Minatoku, Tokyo] 
JORDAN (Al-Mamlaka Al-Haschimija Al-Urdunija; Hashemite Kingdom of Jordan)

Ministry of Education [Amman]

Jordan National Commission for UNESCo [c/o Ministry for Foreign Affairs, Amman]

KENYA (Jamhuri ya Kenya; Republic of Kenya)

Ministry of National Education [Nairobi]

Kenya National Commission for UNESCO [c/o Ministry of National Education, Nairobi]

ISC. / IUS. Students' Union, The University College [P.O.Box 30197 , Nairobi]

Pax Romana [c/o Department of English, University College, P.O.Box 30197, Nairobi]

ISMUN. Kenya United Nations Student Association [P.O.Box 2909, Nairobi]

\section{KOREA}

REPUBLIC OF KOREA

(Han Guk)

Ministry of Education [Seoul]

Korean National Commission for UNESCo [P.O.Box Central 64, Seoul]

World University Service [18 Mook Jung Dong, Choong-ku, Seoul]. - Chairman: Dr. Myung Sun Kim. - Secr.: Bong Sam Kim.

The Korean Federation of Education Associations (1947) [25, 1-ka Sinmun-ro, Chongro-ku, Seoul]. - Pres.: Dr. Louise Yim. - Secr. Gen.: Tai S Chung. - P: The New Education.

IFUW. Korean Association of University Women [132-2 Sungbuk-dong, Seoul] WSCF. Korean Student Christian Council [I.P.O.Box 1884, Seoul]

Pax Romana [No 1-2ka Myong-dong-Chung-ku, Seoul]

IFUW. Korean Association of University Women [132-2 Sungbuk-dong, Seoul] ISMUN. United Nations Student Association Korea [5-2 ka Namdaimcoon Ro, Cheoug Gu, Seoul]. - Pres.: Chun Kya Park.

\section{DEMOCRATIC PEOPLE'S REPUBLIC OF KOREA}

(Tschosen mintschu tschui-immin Konghwa-Guk)

Ministry of Higher Education [Pyongyang]

IUS. Korean Students' Committee [Sarochung, Pyongyang]

KUWAIT (Dawlat al Kuwait)

Ministry of Education [Kuwait]

National Commission of Kuwalt for UNESCo [c/o Ministry of Education Kuwait]

LAOS (Royaume du Laos)

Ministère de 1'Education Nationale, des Beaux-Arts et des Sports-Jeunesse [Vientiane] 
Commission Nationale du Laos pour l'UNESco [c/o Ministère de l'Éducation Nationale, des Beaux-Arts et des Sports-Jeunesse, Vientiane]

Pax Romana. Catholic Students Society [Mission Catholique, Vientiane]

LEEWARD ISLANDS (Federation of Atlantic and Caribbean Territories of United Kingdom)

Department of Education, Montserrat [Montserrat]

Inspector of Schools of St. Kitts, Nevis and Anguilla [St. Kitts]

Virgin Islands Education Department [Tortola]

\section{LESHOTO}

Department of Education [Maseru]

World University Service [University of Botswana, Lesotho and Swaziland, P.O. Roma, via Maseru]. - Chairman: A. Maruping. - Secr.: J.R. Crawford.

WSCF. Student Christian Movement in Lesotho [University of Botswana, Lesotho and Swaziland, P.O. Roma, via Maseru]

Pax Romana. Catholic Student Society [University College, P.O. Roma, via Maseru]

LIBAN (Al-Dschumhūrija Al-Lubnānija)

Ministère de l'Éducation Nationale et des Beaux-Arts [Beyrouth]

Commission Nationale Libanaise pour l'UNESCo [Cité de l'UNESCO, Beym. routh]

IUS. / ISC. Union Nationale des Universitaires du Liban [B.P. 3473, Beyrouth]

IFUW. Association des Libanaises Diplómées des Universités [c/0 Mlle $V$. Saad, B.P. 252, Beyrouth]. - Pres.: Mlle V. Saad. - Prés. (Rélations internat.): Mile H. Douéhi.

WSCF. Orthodox Youth Movement [Rebels Building, Makhoul Street, Beyrouth]

Pax Romana. Union Catholique des Intellectuels du Liban [c/o Prés.: Prof. Bichara Tabbah; 30, Rue Justinien, Beyrouth]

LIBERIA (Republic of Liberia)

Department of Public Instruction [Monrovia]

Liberian National Commission for UNESCo [c/o Department of Public Instruction, Monrovia]

IUS. Liberian National Students Union [P.O.Box 782, Lynch Street 119, Monrovia]

WSCF. National Student Christian Council of Liberia [YMCA Building, Broad Street, Monrovia]

Pax Romana. Newman Society [Catholic Mission, Monrovia]

LIBYA (Al Mamlakah al Libiyya)

Ministry of Education [EI-Beida]

Libyan National Commission for UNESCO [c/o Ministry of Education, elBeida] 
MADAGASCAR (Repoblika Malagasy; République Malgache)

Ministère de l'Éducation Nationale [Tananarive]

Ministère des Affaires Culturelles [Tananarive]

Commission Nationale pour l'UNESCo [Ministère de l'Éducation Nationale, Tananarive]

ISC. Fédération des Associations d'Ettudiants de Madagascar [Campus Universitaire, Ankatso, B.P. 354, Tananarive]

IUS. Fédération des Associations d'Étudiants de Madagascar [Campus Universitaire, Antananarino, B.P. 3004, Tananarive]

WSCF. Fédération Malgache des Étudiants Chrétiens [B.P. 771, Tananarive]. Prés.: Henri Rahaingoson.

Pax Romana. Association Catholique des Universitaires de Madagascar [Antanimena, Tananarivel. - Prés.: Jean Marius Rand ranto.

MALAWI (Republic of Malawi)

Ministry of Education [P.O.Box 50, Zomba]

World University Service [c/o Prof. J.E.G. Utting, University of Malawi, P.O.Box 200, Limbe]

WSCF. Student Christian Organization of Malawi [P.O.Box 183, Lilongwe]

Pax Romana. Catholic Student Society [P.O.Box 368, Limbe]

MALI (République du Mali)

Ministère de l'Éducation Nationale [B.P. 119, Bamako]

Commission Nationale Malienne pour l'UNESCO [c/o Ministère de l'Éducation Nationale, B.P. 119, Bamako]

\section{MALAYSIA (Federation of Malaysia)}

Ministry of Education [Federal House, Kuala Lumpur]

National Commission for UNESCo [Federal House, Kuala Lumpur]

World University Service [1715 Guillermard Road, Kuala Lumpur]. - Chairman: Prof. C.J. Eliezer. - Secr.: Dr. V. Ganeshan.

ISC. Persatuan Kebangsaan Pelajar 2, Malaya [Union House, University of Malaya, Pantal Valley, Kuala Lumpur]. - Pres.: R. Raj Ku mar. - Secr. Gen.: N. Paramsothi.

ISMUN. National Union of Malayslan University Students [Union House, University of Malaya, Pantal Valley, Kuala Lumpur]

Pax Romana [Pope John Centre, Selangor Mansions of Malay Street, Kuala Lumpur]

\section{MAROC / MARRUECOS (El-Maghrib El-Aksa)}

Ministère de l'Éducation Nationale, des Beaux-Arts, de la Jeunesse et des Sports, Direction des Affaires Cuiturelles [Rabat]

Commission Nationale Marocaine pour l'Éducation, la Science et la Culture [Rectorat de l'Université Mohammed V, Avenue Moulay Ali Chérif, Rabat]

IUS. Union Nationale des Etudiants du Maroc [23, Rue Lavoisier, Rabat] 


\section{MARTINIQUE}

Inspection Académique de la Martinique, Service de l'Organisation Scolaire [OK, 300 route Schoelcher, Fort-de-France]

MAURITANIA (Al Jumhurija Al Muslemija Al Mauritanija; République Islamique de Mauritanie)

Ministère de l'Éducation [Nouakchott]

\section{MAURITIUS}

Ministry of Education and Cultural Affairs [Government House, Port Louis]

$$
\text { MÉXICo (Estados Unidos Mexicanos) }
$$

Ministerio de Educación Pública [México, D.F.]

Consejo Nacional Consultivo del Gobierno de México (Comisión Nacional ante la UNESCO) [c/o Ministerio de Educación Pública, México, D.F.]

Di rección General de Asuntos Internacionales de Educación (1960) [Edificio de la Secretaria de Educación Pública, Avenida República Argentina y Luis González Obregón, México, D.F.]. - Dir.: Dr. Emmanuel Pa lacios.

Sociedad de Educación [Sección Educacional, Edificio del Banco de Londres y México, Desp. 7/8, México, D.F.]

IFUW. Asociación de Universitarias Mexicanas [La Otra Banda 51, Villa Obregón, México 20, D.F.]. - Pres.: Dr. M. Chávez de Valasquez, Pres. (Relaciones Internac.): M.C.V. de Arias.

Asociación Nacional de Universidades e Institutos de Enseñanza Superior [Ciudad Universitaria, México 20, D.F.]

IAUPL. Asociation de Profesores Universitarios de México A.C. [Justo Sierra No. 7-Desp. 104, México 1, D.F.]

ISMUN. CEM U [Lic. Grajales Robles 3, Col. del Valle, México 12, D.F.]

ISC. Confederación Nacional de Estudiantes [Apartado postal 541, México, D.F.]

IUS. Confederación de Jóvenes Mexicanos, Sección Estudiantil] [Plaza de Buena Vista 2-412, México 3, D. F.]

WSCF. Movimento Estudiantll Cristlano de México [Avenida Chapultepec 471, México 6, D.F.]

Pax Romana [Tabasco 200, México 7, D.F.]

MOçAMBique (Africa Orientál Portuguesa)

Direcção Provincial dos Serviços de Educação da’ Provincia de Moçambique [Lourenço Marques]

\section{MONGOLIA (Bügd Nairamdach Mongol Ard Uls)}

Ministry of Education [Ulan Bator]

National Commission of Mongolia for UNESCO [c/o Ministry of Foreign Affairs, Ulan Bator]

IUS. Union of Mongolian Students [27 Small Ring Road, Ulan Bator]

NEPAL (Sri Nepála Sarkár)

Ministry of Education [Katmandu]

Nepalese National Commission for UNESCO [c/o Ministry of Education, Katmandu] 
Nepal College and University Teachers' Association [Saraswati Sadan, Katmandu]. - Pres.: B.C. Mall. - Secr.: D.P. Nepali.

World University Service [Dilli Bazar 21/522, Katmandu]. - Chairman: Prof. Prakash Bahadur.

\section{NEW ZEALAND}

Department of Education [Wellington]

New Zealand Council for Educational Research [Education House, 178-182 Willis Street, Wellington C. 2, Post Box 3237, Wellington]. - Dir.: G.W. Parkyn.

Maorl Education Foundation (1961) [c/o Department of Island Territories, Social Development Section, Wellington]

University Grants Committee (1961) [c/o University House, Cnr Bowen and Mowbray Streets, P.O.Box 8035, Wellington]. - Chairman: A.J. Danks.

The New Zealand Vice-Chancellors' Committee (1961) [c/o P.O.Box 196, Wellington]. - Chairman: Dr. J. Williams. - Secr.: B.P. Hampton.

New Zealand National Commission for UNESCO [c/o Department of Education, Wellington]

World University Service [c/o Department of Chemistry, University of Otago, P.O.Box 56, Dunedin]. - Chairman: Prof. H.N. Parton. - Secr.: Dr. Geoffrey Málcolm.

IFUW. New Zealand Federation of University Women [17 Tuhaere Street, Orakei, Auckland E. 1]. - Pres.: Mrs. W.F. Winstone.

ISC. New Zealand Unlversity Students' Association [P.O.Box 6397, Te Aro, Wellington C. 3]

WSCF. New Zealand Student Christian Movement [Box 742, Wellington C. 1].

Pax Romana. University Catholic Society of New Zealand [P.O.Box 2253, Wellington C. 1]

NGWANA s. Swaziland

NICARAGUA (República de Nicaragua)

Ministerio de Educación Pública [Managua]

Comisión Nacional Nicaragüense de Cooperación con la UNESCO [c/o Ministerio de Educación Pública, Managua]

IFUW. Asociación Nicaragüense de Universitarias. - Pres.: Dr. A.B. de Sotomayor [Callejón Riguero, entre Avenida Central y Primera Avenida S.O., Managua], - Secr.: Dr. Amantina M. de Quintanilla]

WUS. Servicio Universitario de Nicaragua [c/o Universidad Nacional de Nicaragua, León]. - Pres.: Dr. Carlos Tunnermann Bernheim. - Secr. Gen.: Br. Jorge Codoy.

ISC. / IUS. Centro Universitario de la Universidad Nacional [Universidad Nacional de Nicaragua, León]

WSCF. Movimento Estudiantil Cristiano de Nicaragua [Apartado 607, Managua]

Pax Romana. Acción Católica Universitaria Nicaraguiense [c/o Alizia Rizo, Apartado 1752, Managua]

NIGER (République du Niger)

Ministère de l'Éducation Nationale [c/o Inspection Académique, Niamey] 
NIGERIA (Federation of Nigeria)

Federal Ministry of Education [Lagos]

National Universities Commission [Cabinet Office, Lagos]

Vice-Chancellors' Committee [c/o Oil Mill Street, Lagos]

National Commission of Nigeria for UNESCO [c/o Federal Ministry of Education, Lagos]

World University Service [c/o Medical School, University of Lagos]. - Chairman: Prof. G.L. Monekosso. - Secr.: Kola Danisa.

ISC. / IUS. National Union of Nigerian Students [University of Ife, Ibadan]

WSCF. Student Christian Movement of Nigeria [10 Awosika Avenue, Bodija Estate, P.O.Box 4014, Ibadan]

Pax Romana [c/o J.U. Obidegwu, Ministry of Commerce and Industry, Enugu]

ISMUN. All Nigeria United Nations Student Association [Ademola Hall, Univerity of Ife, Ibadan]

\section{NEW CALEDONIA (Nouvelle Calédonie)}

Service de l'Instruction Publique, Secteur de l'Éducation, de la Jeunesse et des Sports [Nouméa]

PACIFIC ISLANDS (Trust Territory of the U.S.A.:

Yap, Palau, Marianas Islands, Truk, Ponape, and the Marshalls)

Department of Education in the Trust Territory of the Pacific Islands [Education Office, Agaña Heights, Guam]

PAKISTAN (Islamic Republic of Pakistan)

Ministry of Education [Islamabad]

All-Pakistan Educational Conference (1951) [Saceda Manzil, Nazimabad, Karachi]. - Pres.: Major Shamsuddin Mohamed. - Secr.: Syed Altaf All Brelvi. - P: Al-Ilm-Urdu.

West Pakistan Bureau of Education (1958) [Lahore]. - Dir.: Dr. Abdur Rauf. - P: Statistical Bulletins; Bibliographies and Directories.

Inter University Board of Pakistan (1948) [Islamabad]. - Chairman: Dr. S.D. Choudhuri. - Secr.: Prof. Khwaja Manzoor Hosain.

Standing Committee of Vice-Chancellors [c/o Inter-University Board of Pakistan, Islamabad,

National Commission for UNESCO [c/o Ministry of Education, Islamabad]

World University Service [c/o Dr. Hamid Mahmood, Dep. of Zoology, University of Katachi, Karachi 32]. - Chairman: Dr. I.H. Quresh i.

IAUPL. Pakistan Association of University Professors and Lecturers [University of the Punjab, New Campus, Lahore]. - Secr.: Prof. Kazu S. Ahmad.

IFUW. Pakistan Federation of University Women [Karachi College for Women, Princess Street, Karachi]. - Pres.: Mrs. Z. Rashid A h med.

WSCF. Student Christian Movement in Pakistan [P.O.Box 81, Murray College, Sialkot 1] 
Pax Romana [St. Patrick's College, Saddar, Karachi 3,]

ISMUN. Pakistan United Nations Student Association [Plot No. 1, Row No. 8, 4F Nazimabad, Karachi 18]. - Pres.: Safdar Barlas.

PANAMA (República de Panamá)

Ministerio de Educación [Apartado 3435, Panamá]

Comisión Nacional de Cooperación con la UNESCo [c/o Ministerio de Educación, Apartado 3435, Panamá]

IFUW. Asociación de Mujeres Universitarias de Panamá [Apartado 6920, Panamá]. - Pres.: Dr. R. Barnett.

IUS. Federación de Estudiantes de Panamá [Apartado 3277, Panamá]

Pax Romana [Apartado 52, Avenida Mejico - Centro Católico de Panamá, Panamá]

PAPUA AND NEW GUINEA (Trust Territory of Australia) s. Australia (Commonwealth Office of Education).

\section{PARAGUAY (República del Paraguay)}

Ministerio de Educación y Culto [Chile 849, Asunción]

Comisión Nacional Paraguaya de la UNESCo [c/o Ministerio de Educación y Culto, Chile 849, Asunción]

WUS. Servicio Universitario Mundial [Eligio Ayala 973, Asunción]. - Pres.: Prof. Dr. Amado Adorno.

IFUW. Asociación Paraguaya de Universitarlas Graduadas [c/o C.P.M. Tacuari y 25 de Mayo, Asunción]. - Pres.: Dr. Esther Chirife de Sakoda.

Pax Romana [Olivia 476, Apartado postal 1170, Asunción]

PEOPLE'S REPUBLIC OF CHINA s. China/People's Republic of China

PERỨ (República del Perú)

Ministerio de Educación Pública, Dirección de Organismos Internacionales [Lima]

Comisión Peruana de Cooperación con la UNESCo [c/o Ministerio de Educación Pública, Lima]

Consejo Interuniversitario [c/o Pres.: Dr. Santiago Agusto $C$ alvo, Universidad Nacional de Ingenieria, Lima]

World University Service [c/o Dr. Efrain Morote Best, Universidad Nacional "San Cristobal de Huamanga", Ayacucho]

IFUW. Asociación de Universitarias Graduadas del Perú [Félix Dibos 308, Magdalena del Mar, Lima]. - Pres.: Dr. G. Miranda Quiroz. - Secr. Gen.: Prof. Teresa Pérez.

IUS. Federación de Estudiantes del Perú [Casilla 4931, Lima]

Pax Romana [Apartado postal 3234, Ucayalo 346, Lima]

PHILIPPINES (Republika Ng Pilipinas; República de Filipinas)

Department of Education [Manila]

UNESCO National Commission of the Philippines [1580 Taft Avenue, Manila] 
World University Service [Institute of Student Affairs, 1365, Leon Guinto Street, Ermita, Manila]. - Chairman: Dr. Waldo Perfecto.

Phillppine Association of Colleges and Universities (1932) [Room 227 Shurdut Building, General Luna Street, W.C., Manila]. - Pres.: Dr. Filemon G. Tanchoco.

Association of Christian Schools and Colleges [1648 Taft Avenue, Manila]. Pres.: Juan Nabong.

IFUW. Phllippine Association of University Women [Philippine National Red Cross, United Nations Avenue, Manila]. - Pres.: Geronima T. Pecson. Chairman (Internat. Relations): Pilar H. Li m.

WSCF. Student Christian Movement of the Philippines [Inter Church Center, 1648 Taft Avenue, Manila]. - Secr. Gen.: Nathaniel Cortez.

Pax Romana [3rd Floor, Pius XII Catholic Centre, United Nations Avenue, Manila]

ISMUN. United Nations Student Association - Philippines [Asian Social Institute, 1518 Leon Guinto Street, Ermita, Manila]

FRENCH POL YNESIA (Polynésie Française; Territoire d'Outre-Mer)

Service de l'Enseignement [Papeete]

PUERTo RICo (El Estado Libre y Asociado del Puerto Rico)

Instituto de Cultura Puertorriquefia [Apartado 4184, San Juan]. - Dir.: Ricardo E. Alegria.

U.P.R. Departamento de Actividades Culturales [Universidad de Puerto Rico, Rio Piedras]

Asociación de Maestros de Puerto Rico [Ponce de León Pda. 33, Hato Rey]. Pres.: Maria Arroyo de Colón.

\section{REPÜBLICA DOMINICANA}

Ministerio de Educación, Bellas Artes y Cultos [Santo Domingo]

Comisión Nacional Dominicana de la UNESco [Secretaría de Estado de Educación y Bellas Artes, Santo Domingo]

World University Service [Osvaldo Baer No. 5, Santo Domingo]. - Pres.: Carlos Esteban Deive.

IUS. Federación de Estudiantes Dominicanos [Apartado 294, Santo Domingo] WSCF. Comunidad de Estudiantes Evangélicos [Apartado 727, Santo Domingo] Pax Romana. Movimiento Universitario Dominicano [Apartado 1004, Santo Domingo]

\section{REUNION (Département d'Outre-Mer)}

Direction de l'Éducation Nationale de la Réunion [Saint Denis]

\section{RHODESIA}

Ministry of Education [P.O.Box 8024, Causeway, Salisbury]

World University Service [Manfred Hodson Hall, Private Bag 176 H, Salisbury]. - Chairman: R. Gokal. 
IFUW. Association of University Women of Rhodesia. - Pres.: Mrs. J. Over [40 Orange Grove Drive, Salisbury]. - Chairman (Internat. Relations): Mrs. N.S. Kane.

WSCF. Rhodesia Student Christian Movement [50 Vito Street, P.O. Harare Salisbury]

Pax Romana [Swinton Hall, University College of Rhodesia, Private Bag 176 H, Salisbury]

RWANDA (Republica y'u Rwanda; République Rwandaise)

Ministère de l'Éducation Nationale [Kigali]

Commission Nationale Rwandaise pour l'UNESCo [c/o Ministère de l'Éducation Nationale, Kigali]

Entr'aide Universitaire Mondjale [c/o Prof. Dr. Georges A. Auger, Université Nationale du Rwanda, B.P. 117, Butare]

Pax Romana. Pax Romana Rwanda [B.P. 61, Nyanza]

RYUKYU ISLANDS

Education Department [Naha-City, Okinawa]

ST. HELENA AND DEPENDENCIES

(Atlantic and Caribbean Territories of United Kingdom)

Department of Education [Jonestown]

\section{SALVADOR s. El Salvador}

SAUDI ARABIA (Al-Mamlakah Al'Arabiya As-Sa'udiya)

Ministry of Education [Riyadh]

Saudi Arablan Commission for UNESCo [c/o Ministry of Education, Riyadh]

SENEGAL (République du Sénégal)

Ministère de l'Éducation Nationaie [Dakar]

Commission Nationale du Sénégal pour l'UNEsco [Ministère de l'Éducation Nationale, Dakar]

IUS. Union Générale des Etudiants d'Afrique Occidentale [Citẻ Universitaire, B.P. 234, Dakar-Fann]

WSCF. Association des Etudiants et Elèves Protestants de Dakar [141, Rue de Bayeux, Dakar]. - Secr. gén.: Paul Ou attara.

Pax Romana. Unlon des Etudiants Cathollques de Dakar [Fraternité St. Dominique, Route de Ouakam-km 4, B.P. 5098, Dakar-Fann]. — Prés.: Leopold Eze.

\section{SIERRA LEONE}

Ministry of Education [New England, Freetown]

World University Service [c/o Fourah Bay College, P.O.Box 87, Freetown]

ISC. / IUS. National Union of Sierra Leone Students [Fourah Bay College, P.O. Box 87, Freetown] 
WSCF. Student Christian Movement of Sierra Leone [Cathedral House, P.O. Box 404, Freetown]

Pax Romana. The Catholic Newman Society [Fourah Bay College, P.O.Box 87, Freetown]

ISMUN. Sierra Leone United Nations Student Association [Fourah Bay College, P.O.Box 87, Freetown]

SIKKIM (Protectorate of India)

Department of Education [Gangtok]

SINGAPORE (Republic of Singapore)

Kementerian Pelajaran Singapura (Ministry of Education, Singapore) [Kay Siang Road, Singapore 10]

World University Service [c/o Dr. Z.N. Kadri, 57 College Green, Ducarn Road, Singapore 2]

WSCF. National Student Christian Movement [191 Serangoon Road, Singapore 8]

Pax Romana. Catholic Students' Society of the University of Singapore [73 Bras Basah Road, Singapore 7]

SOLOMON ISLANDS (British Solomon Islands Protectorate)

Department of Education [Honiara]

SOMALIA (Somali Republic; Kharan-ka Somalija; Al-Jumhourija As-Somalija)

Ministry of Education [Mogadisciu]

National Commission of Somalia for UNESCo [c/o Ministry of Education, Mogadisciu]

SOUTH AFRICA (Republiek van Suid Afrika; Republic of South Africa)

Department van'Onderwijs, Kuns en Wetenskap (Department of Education, Arts and Science) [Pretoria, Transvaal]

National Bureau of Educational and Social Research (1929) [c/o Department of Education, Arts and Science, Private Bag 41, Pretoria, Transvaal]. Dir.: Dr. P. M. Robbertse.

The Committee of University Principals (1955) [P.O.Box 1176, Johannesburg, Transvaal]

The Joint Matriculation Board [P.O.Box 392, Pretoria, Transvaal]

World University Service [c/o S.R.G. Office, P.O.Box 375, Pietermaritzburg, Transvaal]. - Chairman: Ian Allen.

The University Teachers' Association of South Africa [University of Cape Town, Private Bag, Rondebosch, C.P.]

IFUW. South African Association of University Women Pres.: Mrs. E. Chaplin [101 Eleventh Avenue, Berea, Durban, Natal]

Pax Romana. National Catholic Federation of Students [P.O.Box 189, Orange Grove, Johannesburg, Transvaal]

WUJS. South African Federation of Student Jewish Assoclations [105 Denhil, 58 Jorissen Street Bramfonten, Johannesburg, Transvaal] 
SUDAN (Jamhuryat Es Sudan; Republic of the Sudan)

Ministry of Education [Khartoum]

National Commission for UNESCO [c/o Ministry of Education, Khartoum]

World University Service [University of Khartoum, P.O.Box 231, Khartoum]. - Chairman: Dr. Mohamed Omer Bashir. - Secr. Gen.: Ahmed Yousif El Tinay.

IFUW. Sudanese Women Graduates Association [c/o Faculty of Arts, University of Khartoum, P.O.Box 231, Khartoum]. - Pres.: Mrs. M.S. Nur.

ISMUN. Sudan United Nations Student Association [Faculty of Economics and Social Studies, University of Khartoum, P.O.Box 231, Khartoum]. Pres.: Abdella Mohammed Abdelrahman.

IUS. Khartoum Unlversity Students' Union [University of Khartoum, P.O. Box 321, Khartoum]

Pax Romana. St. Augustine's Society [c/o Comboni College, P.O.Box 114, Khartoum]. - Pres.: Joseph Bassia Tasa.

\section{SURINAM}

Ministerle van Onderwijs en Volksontwlkkeling (Ministry of Education) [Paramaribo]

Stichting CultureeI Centrum Suriname (Surinam Cultural Centre) (1947) [P.O. Box 1241, Paramaribo]. - Pres.: E.R. Wessels.

\section{SWAZILAND (Ngwana)}

Department of Education [P.O.Box 39, Mbabane]

SYRIE (Al Jamhouriya Al Arabiya As Souriya)

Ministère de l'Éducation [Damas]

Consell Supérieur des Universités [Université de Damas, Damas]

Commission Nationale Culturelle Syrienne pour l'UNESCo [c/o Ministère de l'Éducation, Damas]

Union des Etudiants de l'Université de Damas [Université de Damas, Damas]

TAIWAN s. China/Taiwan

TANZANIA (United Republic of Tanzania)

Ministry of Education [P.O.Box 9121, Dar es Salaam]

Tanzanian National Commission for UNESCo [c/o Ministry of Education, P.O.Box 9121, Dar es Salaam]

World University Service [The University College, P.O.Box 9184, Dar es Salaam]. - Chairman: J.C. D'Souza.

ISMUN [The University College, P.O.Box 9121, Dar es Salaam]. — Secr. Gen.: D.D. N ser eko.

ISC. National Union of Tanzanian Students [The University College, P.O.Box 9184, Dar es Salaam]

IUS. National Union of Tanzanian Students [P.O.Box 15698, Dar es Salaam]

Pax Romana. Dar-es-Salaam University Catholic Students Association [P.O. Box 20603, Dar es Salaam]. - Pres.: Clement Mangana. 
TCHAD (République du Tchad)

Ministère de l'Éducation Nationale [Fort-Lamy]

Commission Nationale Tchadienne pour l'UNESCO [c/0 Ministère de l'Éducation Nationale, Fort-Lamy]

WSCF. Union des Jeunes Chrétiens [B.P. 2138, Fort-Lamy]. - Secr. Gén.: Moussanang Gabriel.

THAILAND (Prades Thai; Muang Thai)

Ministry of Education [Bangkok]

Department of Information [Bangkok]

National Instifute of Culture [Bangkok]

Thailand National Commission for UNESCO [c/o Ministry of Education, Bangkok]

World University Service [1281 Pahol Yothin Highway, Bangkok]. - Chairman: Dr. Kamhaeng Bhalangura.

IFUW. Siamese Association of University Women [4 Soi 26, Sukhumvit Road, Bangkok]. - Chairman (Internat. Relations): Mrs. Somchit Siddhichai.

WSCF. Student Christian Movement of Thailand [328 Phya Thai Road, Bangkok]

Pax Romana [Xavier Hall, 70/9 Rajavithi Road, Bangkok]

TOGo (République Togolaise)

Ministère de l’Éducation Nationale de la République Togolaise [Lomé]

Commission Nationale de la République Togolaise pour 1'UNESCO [c/o Ministère de l'Éducation Nationale, Lomé]

TONGA (Friendly Islands)

Department of Education [Nuku'alofa]

\section{TRINIDAD AND TOBAGO}

Ministry of Education and Culture [Alexandra Street, St. Clair, Port of Spain]

ISC. Regional Union of West Indian Students [c/o Students Guild, University of the West Indies, Faculties of Agriculture and Engineering, St. Augustine]

TSCHAD s. Tchad

TUNISIE (Al Djoumhouria Attunusia; République Tunisienne)

Ministère de l'Éducation Nationale [Tunis]

Commission Nationale Tunisienne pour l'UNESCo [c/o Secrétariat d'État à l'Éducation Nationale, Tunis]

ISC. / IUS. Union Générale des Etudiants de Tunisie [11, Rue d’Espagne, Tunis]

UGANDA (Republic of Uganda)

Ministry of Education [Parliamentary Buildings, P.O.Box 263, Kampala]

Uganda National Commission for UNESCO [c/o Ministry of Education, Parliamentary Buildings, P.O.Box 263, Kampala]

World University Service [Makerere University College, P.O.Box 262, Kampala. - Chairman: Dr. D. Denoon. 
IAUPL. Makerere University College Academic Staff Association [P.O.Box 262, Kampala]. - Secr.: Dr. E.A. Calder.

IFUW. Uganda Association of University Women [P.O.Box 939, Kampala]. Pres.: Mrs. E. Posnansky. - Chairman (Internat. Relations): Dr. M. Welter.

ISC. National Union of Students in Uganda [P.O.Box 16154, Kampala]

IUS. Makerere Students' Guild [Makerere University College, P.O.Box 262, Kampala]

ISMUN. Makerere College United Nations Student Association [New Hall, P.O.Box 262, Kampala]

WSCF. Makerere College Christian Union [Mitchell Hall, P.O.Box 16005, Kampala]

Pax Romana [Makerere University College, P.O.Box 262, Kampala]

UNITED ARAB REPUBLIC

(U.A.R.; Al Jumhurija Al Arabia Al Mutahida)

Central Ministry of Education [33 Sharia Falaky Street, Cairo]

Education Documentation Centre for U.A.R. (1956) [Central Ministry of Education, 33 Sharia Falaky Street, Cairo]. - Dir.: Ibrahim Hafez. - P: Contemporary Trends in Education; Educational Information Bulletin.

New Education Fellowship (N.E.F.), Egyptian Branch (1938) [9 Sharia Kerdasy Street, Cairo]. - Chairman: Dr. Ahmed Abdel Salam El-Kerdany.

Supreme Councll of Universities [University of Cairo, Gizah, Cairo]

United Arab Republic National Commission for UNESCo [Ministry of Education, 17, Shareh Ismail Abu El Fotooh, Dokki, Cairo]

IFUW. Egyptian Association of University Women [26, Chérif Street, Alexandria]. - Pres.: Mrs. D. El Masri. - Secr. (Internat. Relations): Mrs. L. Nasri.

WSCF. Families of Christian University Students [Anba Rueis Building, Ramses Street, Abbasiya, Cairo]

URUGUAY (República Oriental del Uruguay)

Ministerio de Instrucción Pública [Montevideo]

Comisión Nacional de la UNESCo [c/o Ministerio de Instrucción Pública, Montevideo]

IFUW. Asociación de Mujeres Tituladas en la Universidad del Uruguay. Pres.: H. Barreiro [Calle Huidobro 1387, Montevideo]. - Pres. (Relaciones Internac.): I. Goni de Faraldo.

WSCF. Movimiento Estudiantil Cristiano del Uruguay [San José 1457, Montevideo]

Pax Romana [Avenida Rivera 2336-40, Montevideo]

\section{U.S.A. (United States of America)}

Office of Education (1867) [U.S. Department of Health, Education, and Welfare, Washington, D.C. 20525]. - P: Education Directories; Digest of Educational Statistics; Progress of Public Education in the U.S.A.; Catalog of Reports and Studies.

American Councll on Education (1918) [1785 Massachusetts Avenue, N.W., Washington, D.C. 20036]. - Pres.: Dr. Logan Wils on. - P: American Universities and Colleges; American Junior Colleges; The President's Annual Report; The Educational Record; Higher Education and National Affairs; Special Report on Federal Programs; Bulletin on International Education; A Fact Book on Higher Education; Report on Questionnaires. 
National Academy of Education (1965) [c/o Pres.: Dr. Ralph W. Tyler, Center for the Advanced Study of the Behavioral Sciences, 202 Junipero Serra Boulevard, Stanford, California]

College Entrance Examination Board [475 Riverside Drive, New York, N.Y. 10027]. - Pres.: Richard Pearson.

Council for Financial Ald to Education [6 East 45th Street, New York, N.Y. 10017]. - Pres.: Holgar J. Johnson.

National Commission for UNESCO [Bureau of Educational and Cultural Affairs, Department of State, Washington, D.C. 20025]

World University Service [20 West 40th Street, New York, N.Y. 10018]. Chairman: Huston Smith. - Execut. Dir.: John Simons.

Alfred P. Sloan Foundation (1934) [630, Fifth Avenue, New York 20, N.Y.]. Support of projects in education and research. - Pres.: Everett N. Cas e. - Vice-Pres. and Execut. Dir.: Arnold J. Zurcher. - P: Report.

Carnegie Foundation for the Advancement of Teaching (1905) [589, Fifth Avenue, New York, N.Y. 10017]. - Pres.: John W. Gardner. - P: Annual Report.

Ford Foundation (1936) [477 Madison Avenue, New York, N.Y. 10022]. Advancement of human welfare, principally through the support of education. - Chairman of Board: Dr. Julius S trat ton. - Pres.: McGeorge Bundy.

Foundation for Integrative Education (1948) [777 United Nations Plaza, New York, N.Y. 10017]. - Pres.: Kirtley F. Mather. - P: Main Currents in Modern Thought.

The Fund for the Advancement of Education (1951) [477 Madison Avenue, New York, N.Y. 10022.] - Pres.: Clarence H. Faust. - Secr, and Treasurer: John J. Scanlon.

W.K. Kellogg Foundation (1930) [400 North Avenue, Battle Creek, Michigan 49016]. - Administers funds for non-profit agricultural, educational, and health activities in the public interest. - Pres.: Emory W. Morris, Secr.: Leonard L. White. - P: Annual Report.

The Rockefeller Foundation (1913) [111 West 50th Street, New York, N.Y. 10020]. - Grants to universities, research institutes and agencies. Pres.: J. George Harrar. - P: Reviews and Reports.

NEA. National Education Association of the United States [1201, 16th Street, N.W., Washington, D.C. 20036]. - Execut. Secr.: William G. Carr.

Association for Higher Education (1924; 1943). - One of the four original departments of the National Education Association. - Pres.: Stephen J. Wright, President, United Negro College Fund [22 East 54th Street, New York, N.Y. 10022]. - Execut. Secr.: G. Kerry Smith. - P: College and University Bulletin; Current Issues in Higher Education.

Association of American Universitles (1900) [1785 Massachusetts Avenue, N.W., Washington, D.C. 20036]. - Pres.: Grayson Kirk. - Secr.-Treasurer: Nathan M. Pusey. - Execut. Secr.: Charles P. McCurdy jr.

Association of State Colleges and Universities (1961) [1785 Massachusetts Avenue, N.W., Washington, D.C. 20036]. - Exec. Dir.: Allan W. Ostar. - P: Memo to the President.

National Association of State Universities and Land-Grant Colleges (1887) [1785 Massachusetts Avenue, N.W., Washington, D.C. 20036]. - Pres.: Edgar F. Shannon jr. - Execut. Dir.: Russell I.' Thackrey.

Association of American Colleges (1915) [1818 R Street, Northwest, Washington, D.C. 20009]. - Pres.: Richard H. Sullivan. - P: Liberal Education.

Association of Colleges and Secondary Schools [Barber-Scotia College, Concord, North Carolina]. - Pres.: M.H. Estaras. - Secr.: L.S. Cozart.

Association of Urban Universities (1914) [c/o Secr.-Treasurer: Talman W. Van Arsdale jr.; Bradley University, Peoria, Illinois 61606$]$. — Newsletter; Annual Proceedings. 
Association of Governing Boards of Universities and Colleges [1785 Massachusetts Avenue, N.W., Washington, D.C. 20036]. - Execut. Vice-Pres.: J.L. Zwingle.

Middle States Association of Colleges and Secondary Schools [New York University, New York 3, N.Y.]. - Secr.: Elwood C. Kastner.

New England Association of Colleges and Secondary Schools [c/o the Secr.Treasurer: Dana M. Cotton, Graduate School of Education, Harvard University, Cambridge 38, Mass.]

North Central Association of Colleges and Secondary School [5454 South Shore Drive, Chicago 15, 111.]. - Secr.: Norman Burns.

Northwest Association of Secondary and Higher Schools [University of Oregon, Eugene, Oregon]. - Execut. Secr.: F.L. S te ts on.

Southern Association of Colleges and Schools [795 Peachtree Street, Northeast, Atlanta, Georgia]. - Dir.: Felix Robb.

Southern Regional Education Board [130 Sixth Street, N.W., Atlanta 13, Georgia]. - Dir.: Winfred L. Godwin.

Western College Association [Mills College, Oakland, California]. - Execut. Secr.: Francis H. Herrick.

Western Interstate Commission for Higher Education [University East Campus, 30th Street, University of Colorado, Boulder, Colorado]. - Execut. Dir. Robert H. Kroepsch.

New England Board of Higher Education [31 Church Street, Winchester, Mass.]. - Execut. Secr.: Martin Lichterman.

Near East College Association, Inc. [548 Fifth Avenue, New York;36, N.Y.]. Execut. Dir.: Robert S. B rookings.

Association of Collegiate Schools of Architecture [The Octagon, 521, 18th Street, N.W., Washington, D.C. 20036]

National Association of Schools of Art [50 Astor Place, New York 3, N.Y.]. Pres.: Joseph McCullough.

Accrediting Association of Blble Institutes and Blble Colleges [Middle Highway, Barrington, Rhode Island]. - Secr.: Terrelle B. Crum.

American Association of Collegiate Schools of Business [101 North Skinker Road, Station 24, St. Louis 5, Missouri]. - Execut. Secr.: James F. Kan e.

Council of Dental Education - American Dental Association [222 E. Superior Street, New York, N.Y. 10017]. - Secr.: Kenneth Wessele.

American Association of Dental Schools 211 F. Chicago Avenue, Chicago Illinois]. - Secr.: Dale E. Mattson.

American Society for Engineering Education [1346 Connecticut Avenue, N.W. Washington, D.C.]. - Secr.: W. Leighton Collins.

American College Health Association [c/o University of Miami, Coral Gables 46, Florida]. - Secr.-Treasurer: Dr. Ruth B oynton.

Association of Schools of Public Health [School of Public Health, Yale University, 60 College Street, New Haven, Connecticut 06510]. - Pres. Myron E. Wegman.

American Council on Education for Journalism [Indiana University, Bloomington, Indiana 47 405]. - Head: Frank A hlgren.

American Association of Schools and Departments of Journalism [School of Journalism, University of Kansas, Lawrence, Kansas]. - Secr.-Treasurer: Elmer F. Beth.

Association of American Law Schools [1501 New Hampshire Avenue, N.W. Washington, D.C.]. - Execut. Dir.: Michael H. Gordozo.

Association of American Library Schools [c/o Department of Library Science, Catholic University of America, Washington, D.C. 10017]. - Pres. Dorothy Bevis. - Secr.: Rev. James J. Kortendick. 
Association of American Medical Colleges [1501 New Hampshire Avenue N.W., Washington, D.C.]. - Execut. Dir.: Robert C. Berson.

National Association of Schools of Music [1501 New Hampshire Avenue, N.W., Washington, D.C.]. - Execut. Secr.: Warren Scharf.

Council on Optometric Education - American Optometric Association [7000 Chippewa Street, St. Louis, Missouri 63110]. - Chairman: N.E. Abrahamsen sr., O.D.

A merican Association of Osteopathic Colleges [Kirksville College of Osteopathy and Surgery, Kirksvilie, Missouri]. - Secr.: J.D. Denslow.

American Council on Pharmaceutical Education [77 West Washington Street, Chicago 2, Illinois]. - Secr.: Fred T. Mahaffey.

American Association of Colleges of Pharmacy [1507 M Street, N.W., Washington, D.C. 20036]. - Secr.: Charles W. Bliven.

American College Public Relations Association [1785 Massachusetts Avenue, N.W., Washington, D.C. 20036]. - Execut. Vice-Pres.: John W. Leslie.

American Association of Schools of Religious Education [California Baptist Seminary, Covina, California]. - Secr.: Adrian Heat on.

Society for Religion In Higher Education (formerly National Council on Religion in Higher Education) [400 Prospect Street, New Haven, Connecticut]. Execut. Dir.: Lawrence P. de Boer.

Council on Social Work Education [345 E. 46th Street, New York, N.Y. 10017]. - Consultant on Educational Services: Mildred Sikkema.

American Association of Colleges for Teacher Education (NEA) [1201, 16th Street, N.W., Washington, D.C. 20036]. - Execut. Secr.: Edward C. Pomeroy.

American Association of Theological Schools [Third National Bank Building, Dayton 2, Ohio]. - Execut. Dir.: Jesse H. Ziegler.

Association of Summer Session Deans and Directors [University of Colorado, Regent Hall, Boulder, Colorado 80304]. - Head: William A. Owens.

Association of University Evening Colleges [University of Oklahoma, Norman, Oklahoma]. - Dir.: Howell McGee.

National University Extension Association [1820 Massachusetts Avenue, N.W. Washington, D.C. 20036]. - Execut. Dir.: Robert J. Pitchell.

Adult Education Association of the U.S.A. (1951) [1225 Nineteenth Street N.W., Washington, D.C. 20036]. - Execut. Dir.: Eugene I. Johnson. P: Adult Education; Adult Leadership.

American Conference of Academic Deans [Denison University, Granville, Ohio 43023]. - Chairman: Rosemary Pierrel.

IAUPL. American Association of University Professors [1785 Massachusetts Avenue, N.W., Washington, D.C. 20036]. - Secr. Gen.: William P. Fiedler.

National Association of Collegiate Deans and Registrars [Albany State College, Albany, Georgia 31 705]. - Head: E.M. Thorpe.

American Association of College Business Officers [Albany State College, Albany, Georgia]. - Secr.: E.J. Junior jr.

IFUW. American Association of University Women [2401 Virginia Avenue, N.W., Washington, D.C. 20037]. - Pres.: Dr. Blanche H. Dow. Chairman (Internat. Relations): Dr. Alona E. Evans.

ISC. United States National Student Association [2115 S. Street, N.W., Washington, D.C. 20008]

ISMUN. Collegiate Council for the United Nations [345 East 46th Street, New York, N.Y. 10017]. - Dir.: Joan Clark. 
WSCF. National Student Christian Federation [475 Riverside Drive, New York, N.Y. 10027]. - Secr. Gen.: Rev. Leonard Clough.

Pax Romana [1312 Massachusetts Avenue, N.W., Washington, D.C. 20005] WUJS. Student Zionist Organization [515 Park Avenue, New York, N.Y. $10017]$ - [220 South State Street, Chicago, Illinois 60604]

National Association of Foreign Student Affairs [809 United Nations Plaza, New York, N.Y. 10017]. - Secr.: Jeanne Brockmann.

VENEZUELA (República de Venezuela)

Ministerio de Educación [Caracas]

Consejo Nacional de Universidades [c/o Ministerio de Educación, Caracas]

Dirección de Cultura y Bellas Artes del Ministerio de Educación [Este 2 con Sur 25, El Conde, al norte de la Plaza Morelos, Los Caobos, Caracas]. Dir.: Arturo Croce.

Comisión Nacional Venezolana de Cooperación con la UNESCo [c/o Dirección de Polftica Internacional, Ministerio de Relaciones Exteriores, Caracas]

IUS. Federación de Centros Universitarios [Universidad Central de Venezuela, Ciudad Universitaria, Caracas]

WSCF. Movimiento Estudiantil Cristiano de Venezuela [Edificio Viscaya, Apartado B-2, Avenida Las Palmas, Los Caobos, Caracas]

Pax Romana. Movimiento Universitario Católico [Directiva Central Nacional Apartado Este $n^{\circ} 4768$, Caracas]

\section{VIETNAM}

REPUBLIQUE DU VIETNAM (Viet-Nam Cong-Ha)

Ministère de l'Éducation et des Affaires Culturelles [70 Lê-Thanh-Tôn, Saigon] Commission Nationale du Viet-Nam pour l'UNESCo [89 Lê-Van-Duyêt, Saigon]

World University Service [c/o Vietnam WUS House, 11, Hong-Thap-Tu, Saigon]. - Pres.: Prof. Phan Tan Chuc.

Pax Romana [Centre Résurrection, 229, rue Hien-Vuong, Saigon]

\section{RÉPUBLIQUE DÉMOCRATIQUE DU VIETNAM (Viet-Nam Dan-Chu Cong-Hoa)}

Ministère de l'Enseignement Supérieur et Secondaire Spécialisé [Hà-nôi] Syndicat des Enseignants Vietnamiens [Hà-nôi]

IUS. Union Nationale des Étudiants du Viêt-Nam [64 Ba Trieu, Hà-nôi]

$$
\text { WESTERN SAMOA (Samoa i Sisifo) }
$$

Department of Education [Apia]

ZAMBIA (Republic of Zambia)

Ministry of Education [P.O.Box RW. 93, Ridgeway, Lusaka]

World University Service [c/o Fergus Macpherson; University of Zambia, P.O.Box 2379, Lusaka]

ISC. / IUS. National Union of Zambia Students [P.O.Box 1132, Lusaka]

WSCF. Zambia Student Christian Movement [P.O.Box 18, Chisamba]

Pax Romana. Catholic Student Society of Zambia [Oppenheimer College, Lusaka] 


\section{DIE AUSSEREUROPÄISCHEN UNIVERSITÄTEN UND HOCHSCHULEN IN ALPHABETISCHER REIHENFOLGE DER ORTE}


